Supporting Information

\title{
Mitochondria-Targeting Enhanced Phototherapy by Intrinsic Characteristics Engineered "One-for-All” Nanoparticles
}

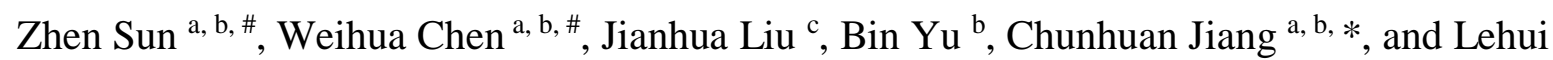
$\mathrm{Lu}^{\mathrm{a}, \mathrm{b}, *}$

${ }^{\text {a }}$ State Key Laboratory of Electroanalytical Chemistry, Changchun

Institute of Applied Chemistry, University of Science and Technology of China, Changchun 130022, P. R. China

${ }^{\mathrm{b}}$ University of Science and Technology of China, Hefei 230026, Anhui, P. R. China

${ }^{c}$ Department of Radiology, Second Hospital of Jilin University, Changchun 130041, P. R. China

*Corresponding author: Chunhuan Jiang, E-mail: chunhuanjiang@ciac.ac.cn

Lehui Lu, E-mail: 1ehuilu@ ciac.ac.cn; Fax: +86-43185262406

\#These authors contributed equally to this work. 


\section{Supplementary Experimental Section}

\section{Materials}

Bismuth (III) neodecanoate, Oleyamine, fluorescein isothiocyanate, calcein-AM, propidium iodide (PI), 3-(4,5-dimethylthiazol-2-yl)-2,5-diphenyltetrazolium bromide (MTT), methyl- $\beta$-cyclodextrin $(\mathrm{m} \beta \mathrm{CD})$ were purchased from Sigma-Aldrich. Polyvinylpyrrolidone (PVP, 8000), sulfur, poloxamer, Cy5.5 NHS ester were obtained from Aladdin. mPEG-NH 2 was purchased from Shanghai Yarebio Technology Co., Ltd. Gelatin and $\mathrm{FeCl}_{2} \cdot 4 \mathrm{H} 2 \mathrm{O}$ were purchased from Tianjin Guangfu Fine Chemical Research Institute. Chlorpromazine hydrochloride (CPZ) and genistein were purchased from J\&K Scientific. Amiloride was purchased from Heowns Biochem LLC. Sodium dodecyl sulfate, 1,3-Diphenylisobenzofuran were purchased from Macklin. Terephthalic acid (TA) was obtained from Alfa aesar. LysoTracker Red DND-99, MitoTracker®Red CMXRos were purchased from Yeasen biotech Co., Ltd. DCFH-DA (reactive oxygen species assay kit) was obtained from Beyotime Biotechnology Co., Ltd. Dulbecco's modified eagle medium (DMEM). All chemicals were analytical grade and used as received without further purification. Water used in the experiment was purified by a Millipore system.

\section{Instruments}

Transmission electron microscope (TEM; Hitachi H 9000 NAR) was used to observe the morphologies. Hydrodynamic diameter and Zeta potential were tested on Zetasizer Nano ZS (Malvern Instruments Ltd., UK). X-ray photoelectron spectroscopy was carried on an HP 5950A X-ray photoelectron spectrometer. UV-vis-NIR spectra were detected on a UV-vis-NIR spectrophotometer (VARIAN CARY 50). UV-visible diffuse reflectance spectra (DRS) were measured by Hitachi U-3900 spectrometer. Fourier transform infrared (FTIR) spectra were collected on a VERTEX 70 FTIR spectrometer (Bruker). Inductively coupled plasma mass spectrometry (ICP-MS) data were collected on an ELAN 9000/DRC system. Fluorescence 
imaging was observed by a Leica fluorescence microscope and Confocal Laser Scanning Microscope (CLSM, Leica TCS SP2, Leica Microsystems, Mannheim, Germany). 
$\mathbf{a}$

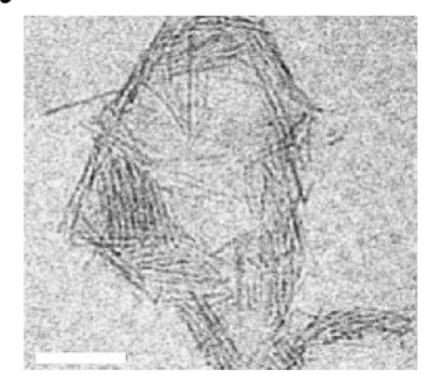

b

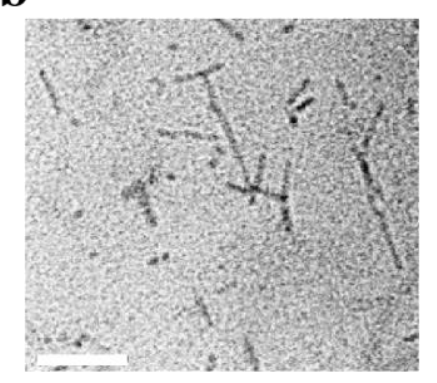

c

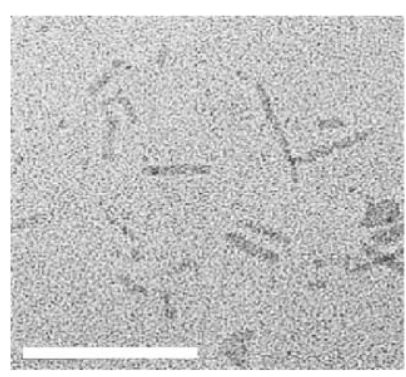

Figure S1. TEM images of (a) 0.5-FeBS, (b) 2-FeBS, (c) 4-FeBS. Scale bar: $50 \mathrm{~nm}$.
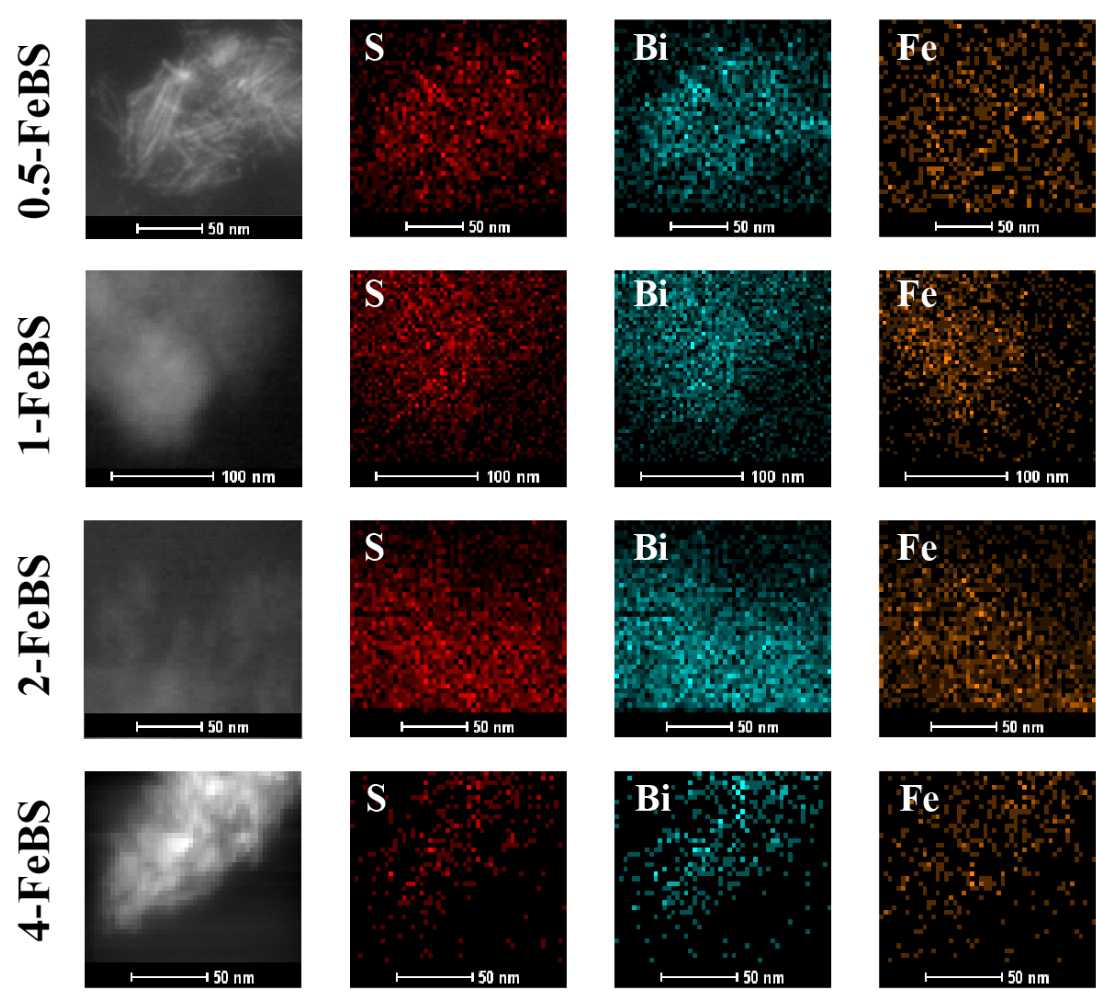

Figure S2. The energy dispersive X-ray elemental mapping of 0.5-FeBS, 1-FeBS, 2-FeBS, and 4-FeBS.

Due to the excessively small width of the material, the imaging of individual nanoparticles is limited by the resolution of instrument. Thus, the nanomaterials in the state of aggregation were selected for capture. 


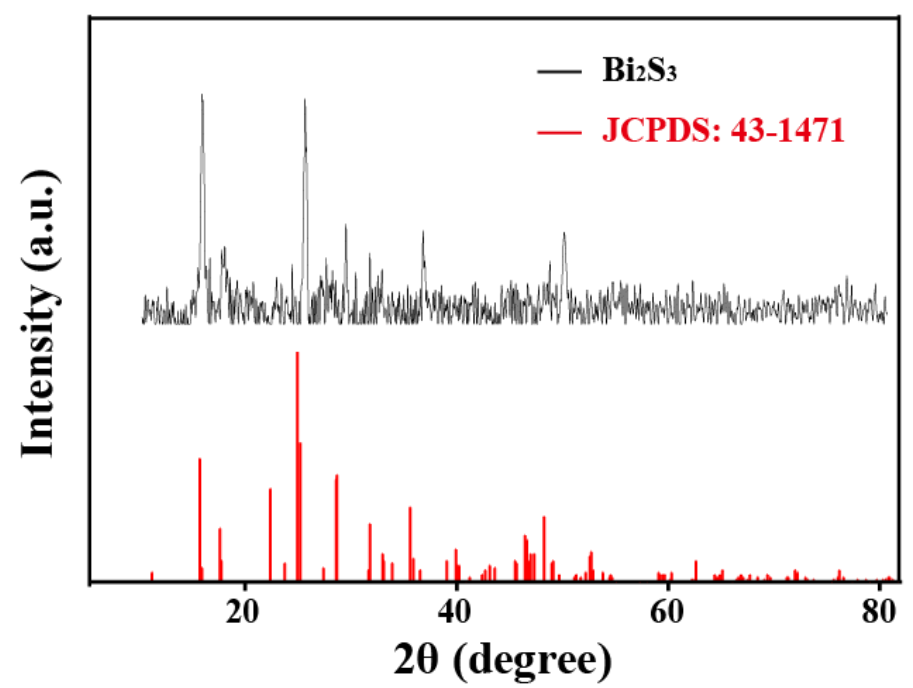

Figure S3. The XRD pattern of $\mathrm{Bi}_{2} \mathrm{~S}_{3}$ before Fe-doping.

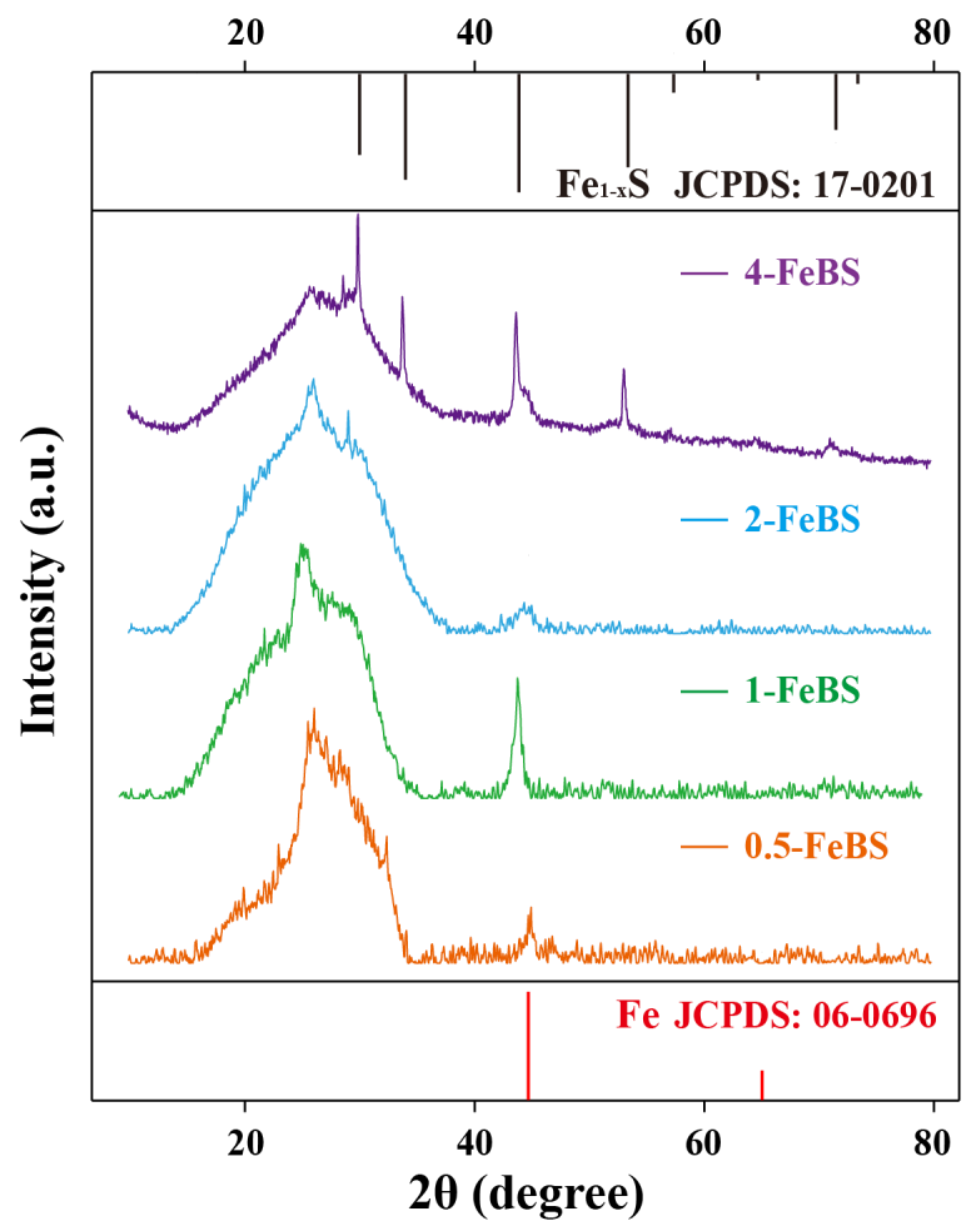

Figure S4. The XRD pattern of 0.5-FeBS, 1-FeBS, 2-FeBS, 4-FeBS. 
a

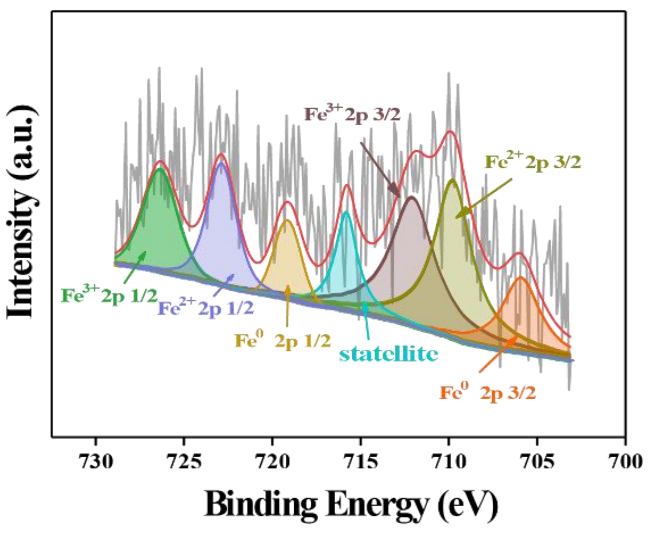

c

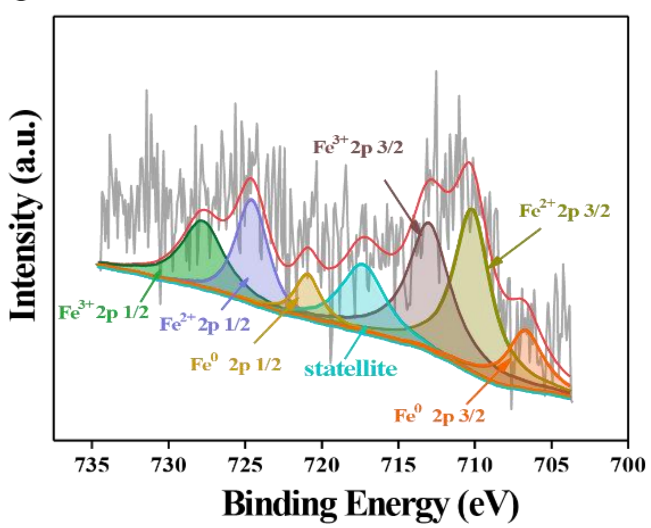

b

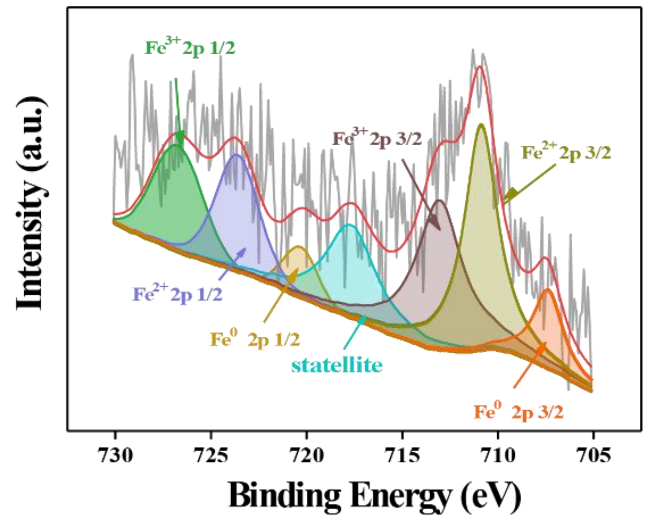

d

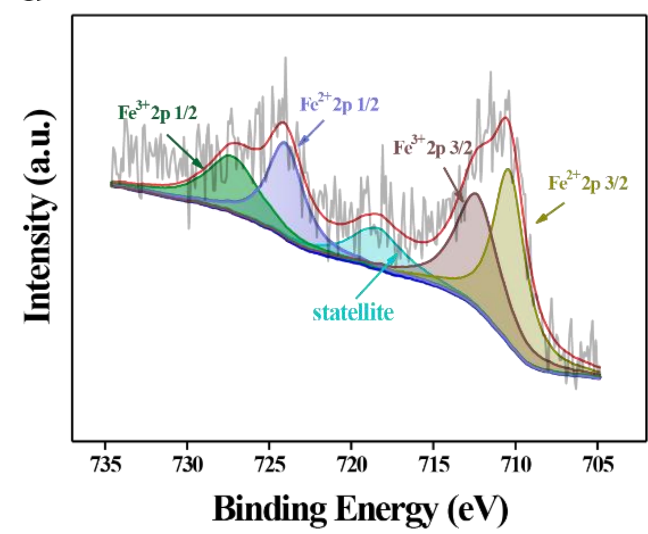

Figure S5. High resolution XPS survey for Fe $2 p$ and $3 p$ and satellite peak of (a) 0.5-FeBS, (b) 1-FeBS, (c) 2-FeBS and (d) 4-FeBS. 
a

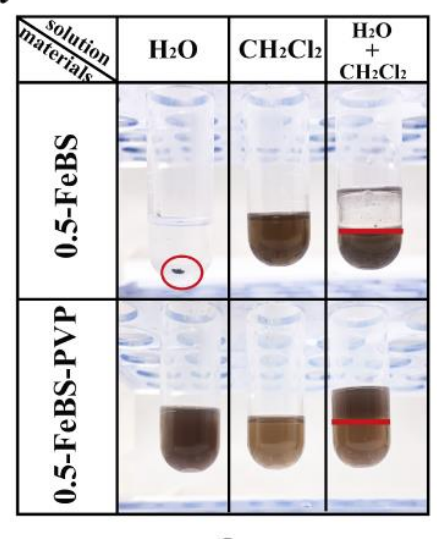

b

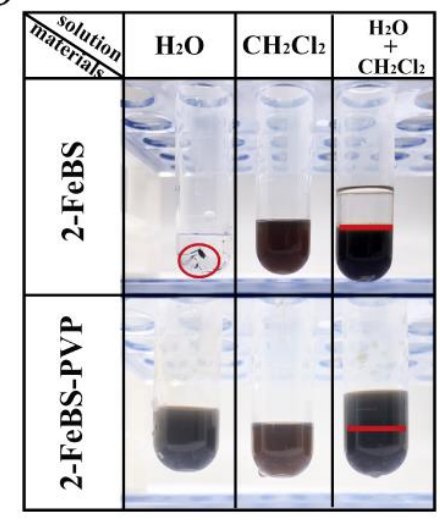

C

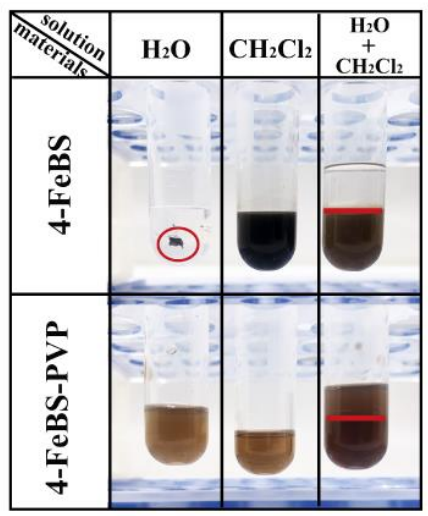

Figure S6. The lipophilicity and hydrophilicity of $x$-FeBS and $x$-FeBS-PVP (a) $x=0.5$, (b) $x=2$, (c) $x=4$. Red circle shows insoluble 1-FeBS in $\mathrm{H}_{2} \mathrm{O}$. Red dotted line represents the immiscible interface of $\mathrm{H}_{2} \mathrm{O}$ and $\mathrm{CH}_{2} \mathrm{Cl}_{2}$.
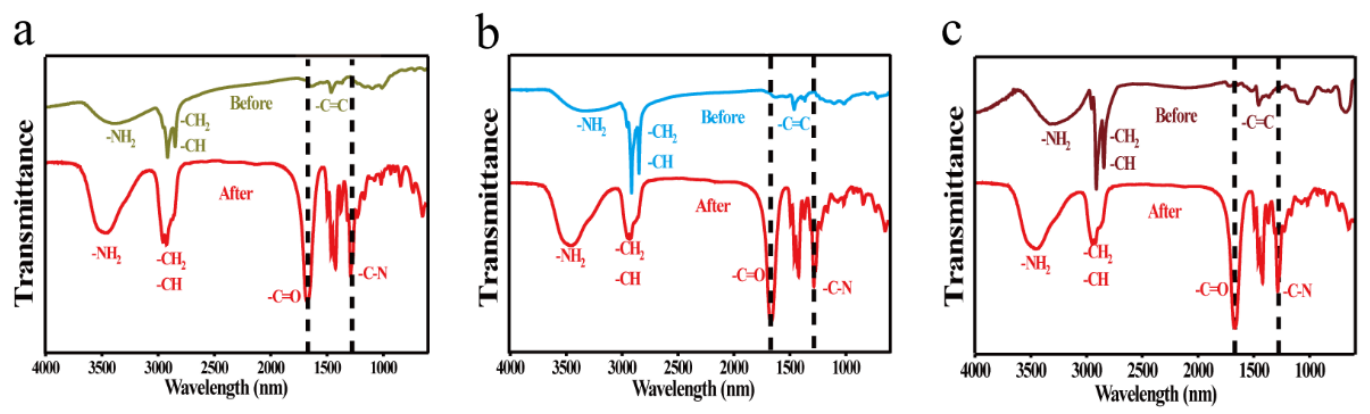

Figure S7. FTIR spectra of (a) 0.5-FeBS-PVP, (b) 2-FeBS-PVP, (c) 4-FeBS-PVP. 


\begin{tabular}{|c|c|c|c|}
\hline Sample & Fe $(\mu \mathrm{g} / \mathrm{mL})$ & Bi $(\mu \mathrm{g} / \mathrm{mL})$ & $\mathrm{S}(\mu \mathrm{g} / \mathrm{mL})$ \\
\hline 0.5-FeBS-PVP & 0.4736 & 33.316 & 6.872 \\
\hline 1-FeBS-PVP & 1.69 & 22.86 & 5.116 \\
\hline 2-FeBS-PVP & 7 & 15.79 & 6.76 \\
\hline 4-FeBS-PVP & 8.01 & 21.748 & 8.04 \\
\hline
\end{tabular}

Figure S8. The quantitative doping level of Fe in $1 \mathrm{mg} / \mathrm{mL}$ of different samples.

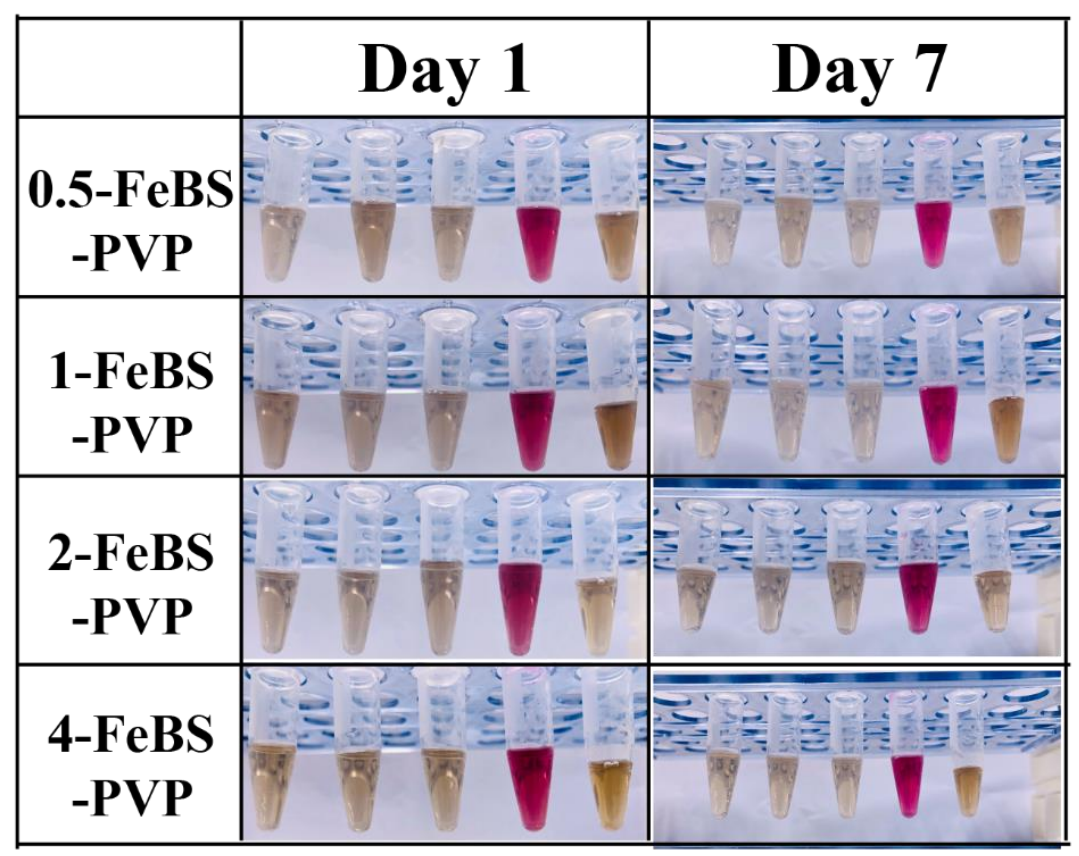

Figure S9. The long-term stability of nanoparticles in different solutions. From left to right: water, PBS, saline, DMEM, serrm. 

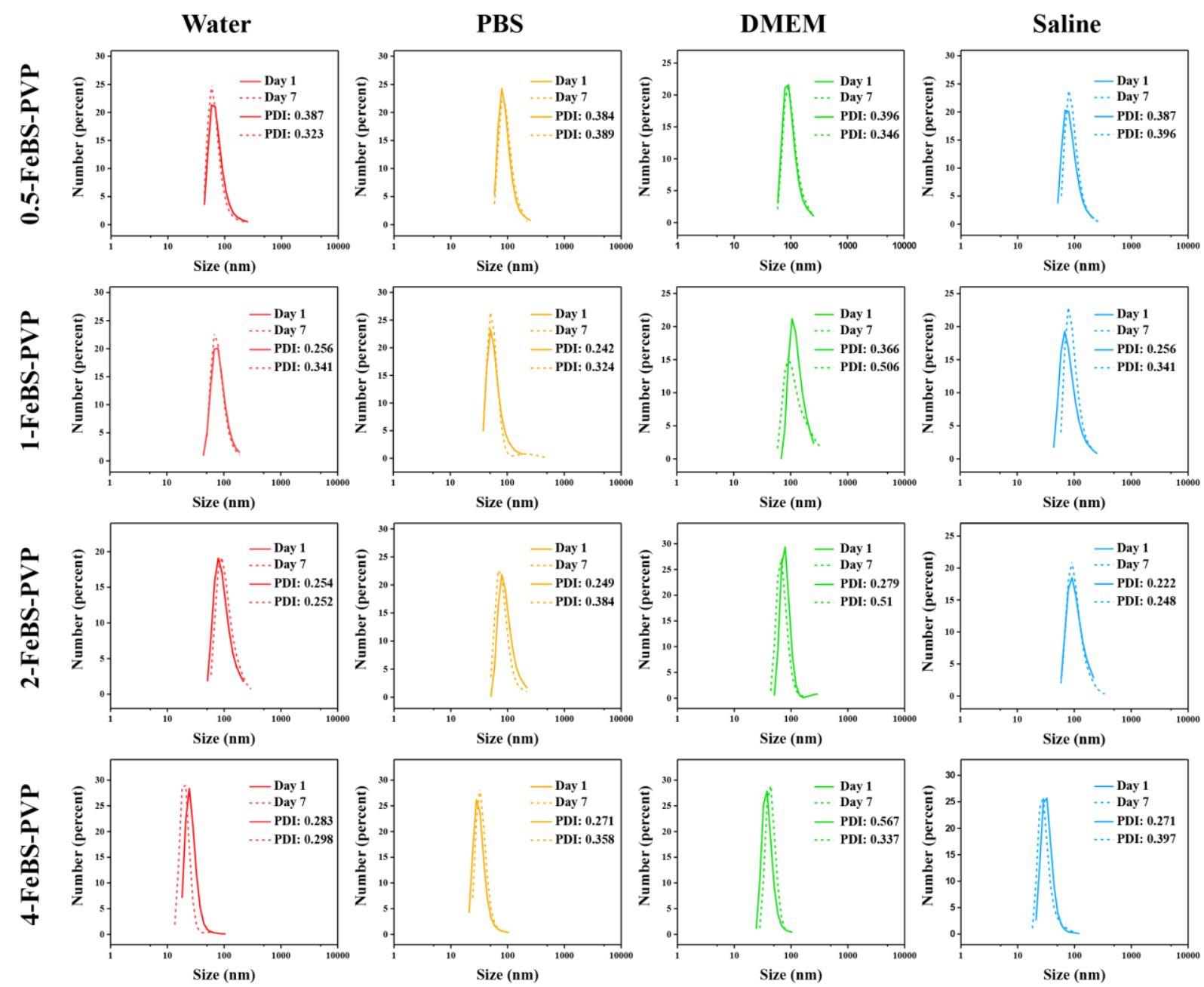

Figure S10. The long-term stability and polydispersity index (PDI) of nanoparticles were measured by DLS. 

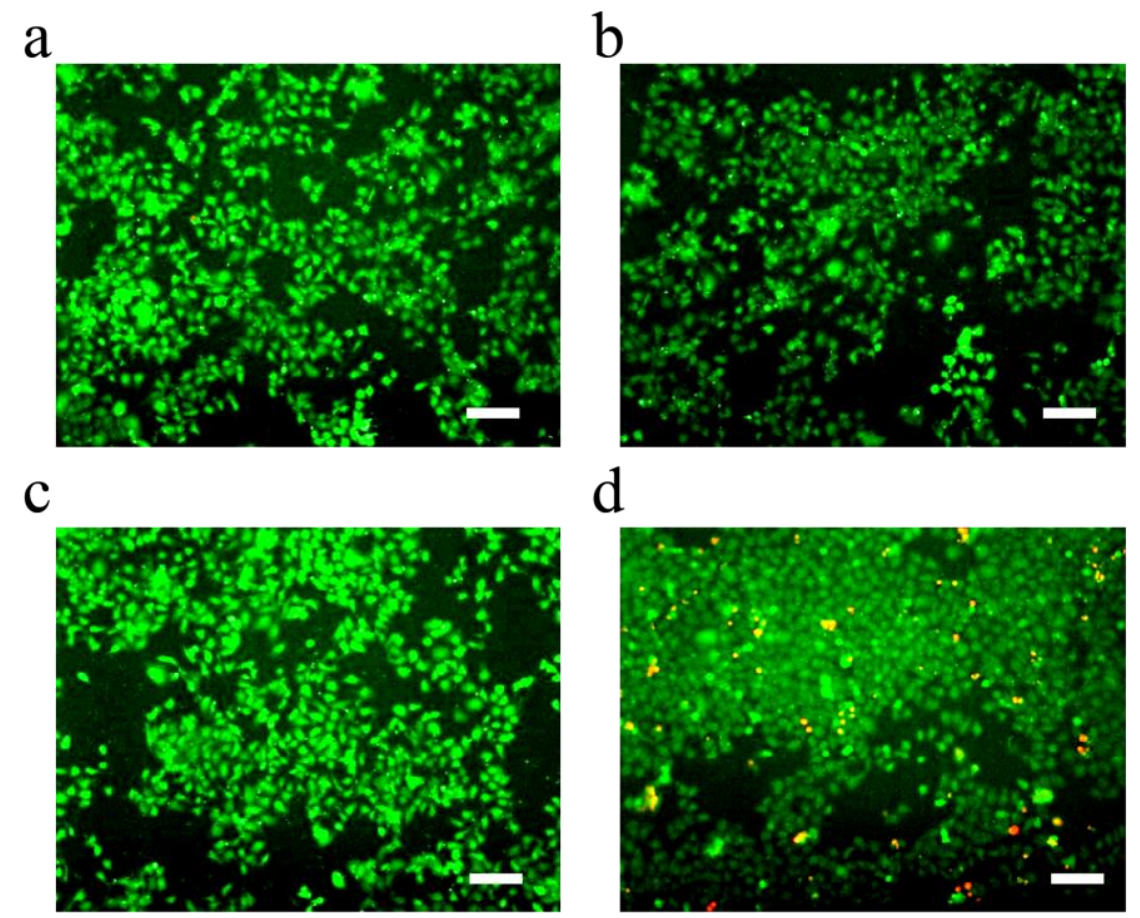

d

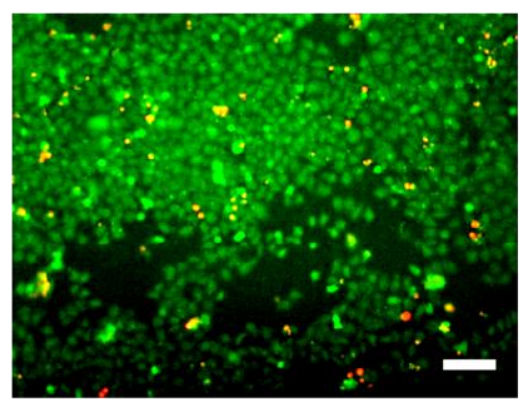

Figure S11. Fluorescence imaging of calcein AM (green)/PI (red) co-stained HeLa cells coincubated with (a) 0.5-FeBS-PVP, (b) 1-FeBS-PVP, (c) 2-FeBS-PVP, (d) 4-FeBS-PVP at 100 $\mu \mathrm{g} / \mathrm{mL}$. Scale bar: $250 \mu \mathrm{m}$. 

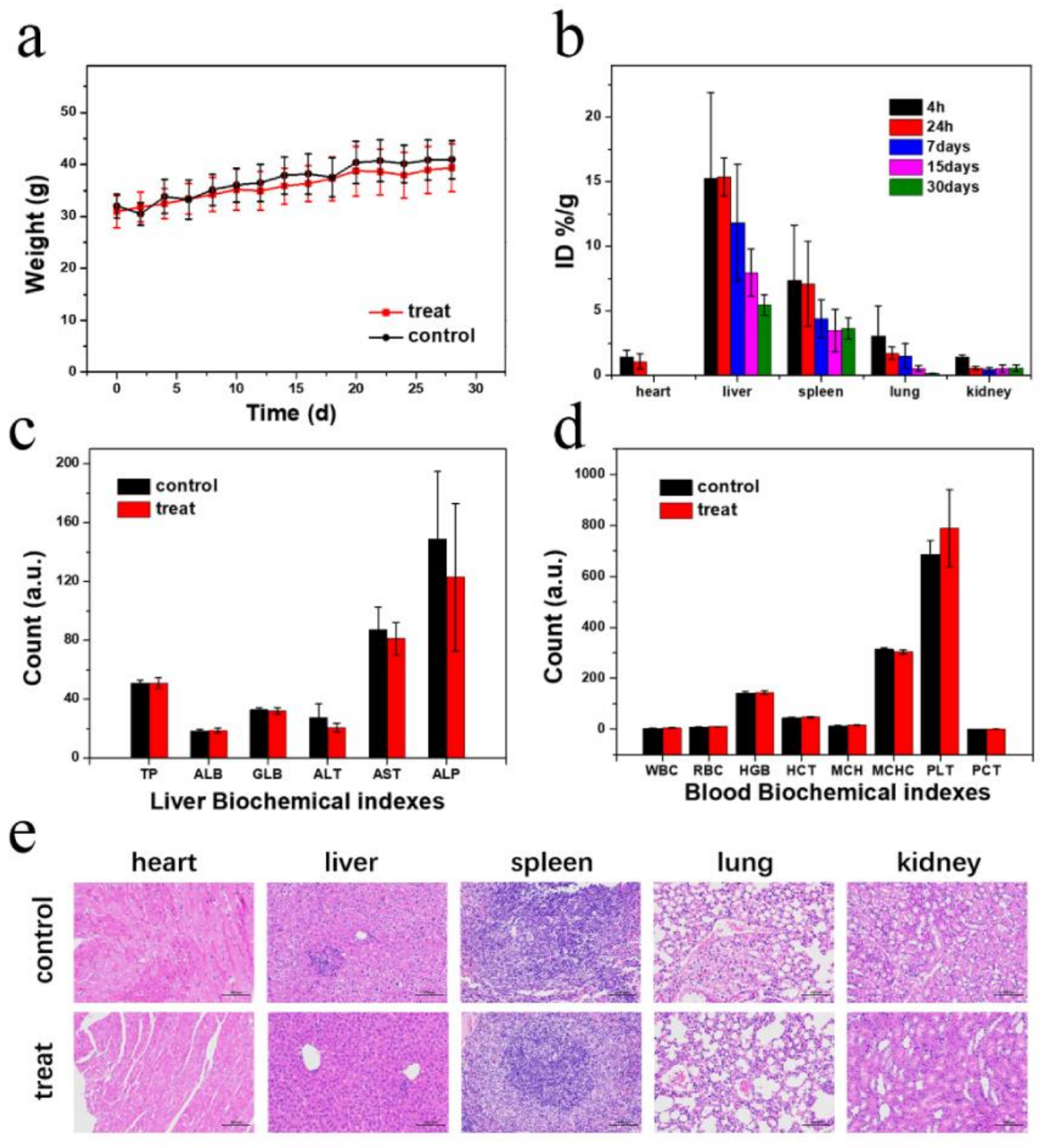

Figure S12. In vivo biocompatibility of 1-FeBS-PVP after intravenous injection with $\mathrm{Bi}^{3+}: 15$ $\mathrm{mg} / \mathrm{kg}$. (a) The changes of body weight in 30 days. (b) The distribution of $\mathrm{Bi}^{3+}$ in major organs (heart, liver, spleen, lung and kidney). (c) Liver biochemical assay (n=6). (d) Blood hematology analysis $(n=6)$. (e) The H\&E stained tissue slices on the thirtieth day. 

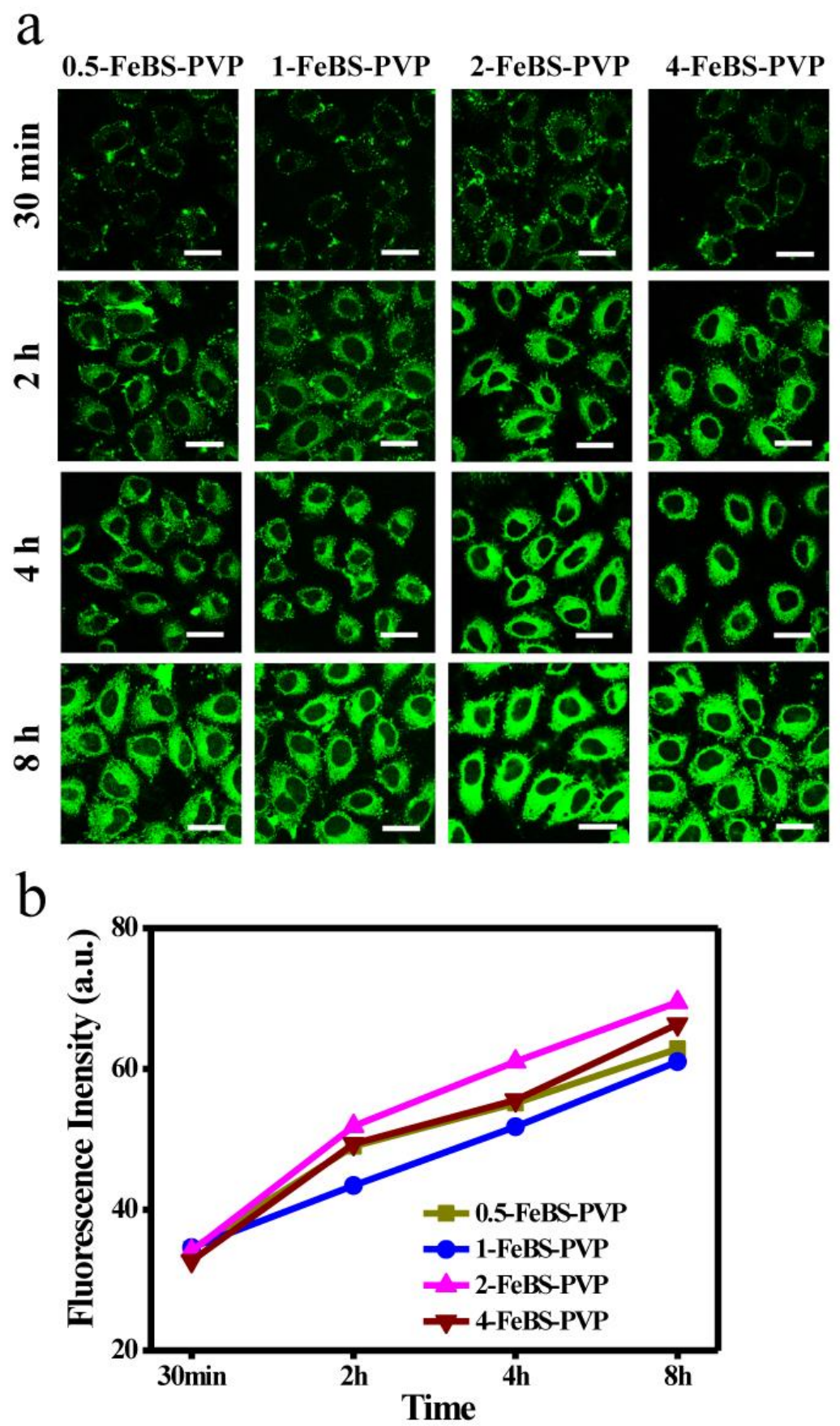

Figure S13. (a) CLSM images of HeLa cells, co-incubated with FITC labeled $x$-FeBS-PVP for different times at $100 \mathrm{ppm}$. Scale bar: $30 \mu \mathrm{m}$. (b) FL intensity changes in HeLa cells. With the increase of incubation time, the intracellular fluorescence intensity, that is, the cell's uptake of materials is significantly enhanced. 


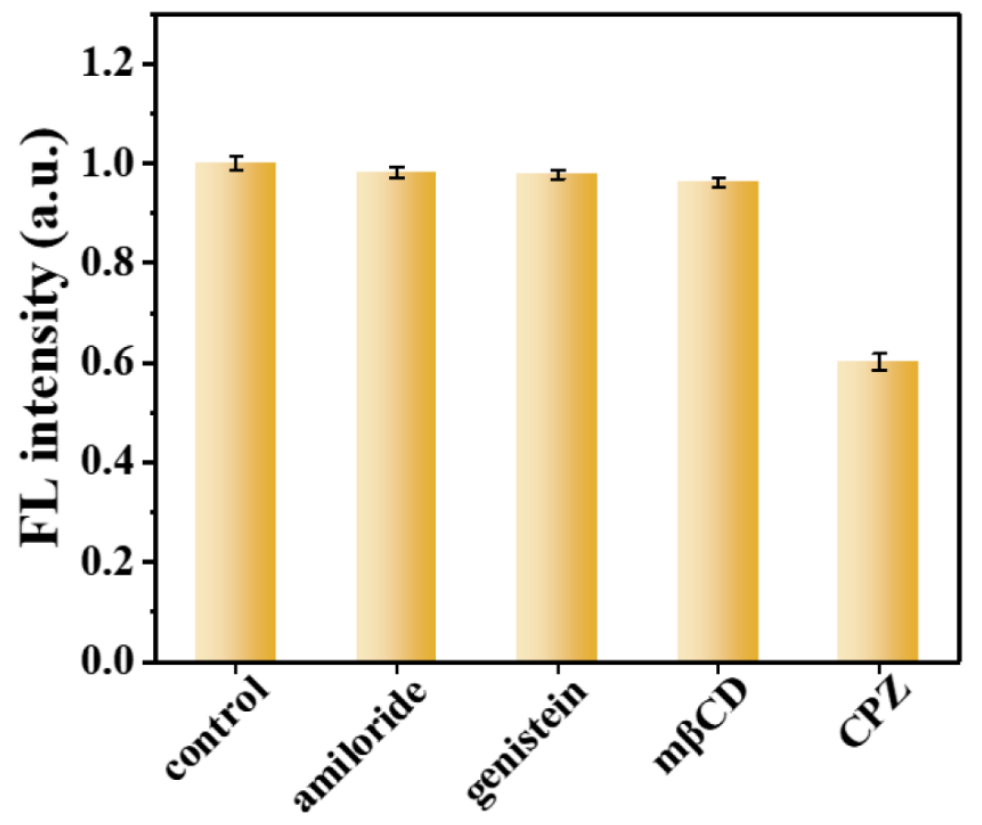

Figure S14. Cellular internalization mechanism of 1-FeBS-PVP in HeLa cells. The decreased FL intensity suggested that cell entry was inhibited. Error bars indicate standard deviation $(n=4)$. 

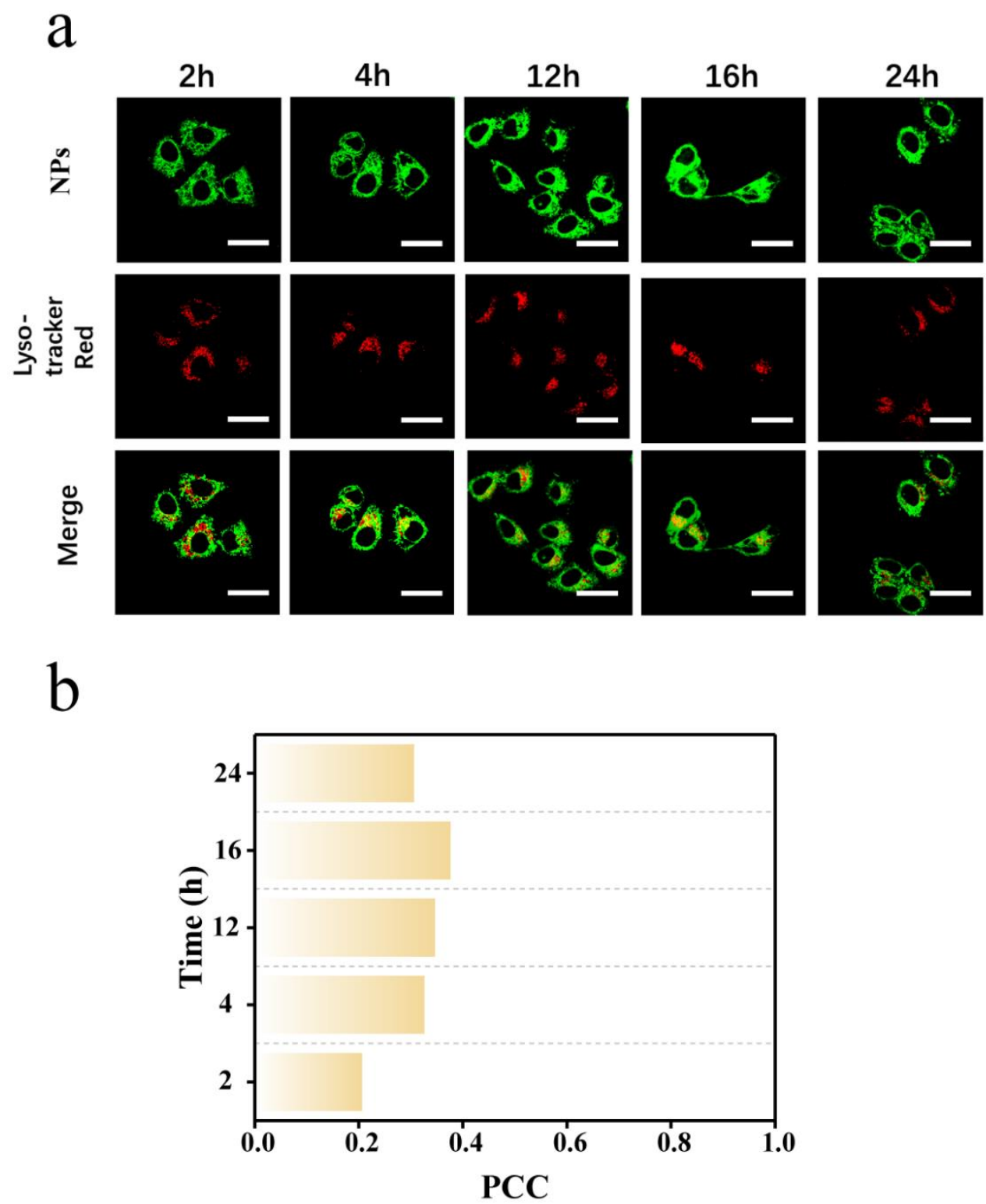

Figure S15. (a) Confocal images of HeLa cells after incubated with FITC labeled 1-FeBS-PVP colocalized with commercially lysosomes tracker at different times. Scale bar: $30 \mu \mathrm{m}$. (b) The PCC value of various groups in (a). 

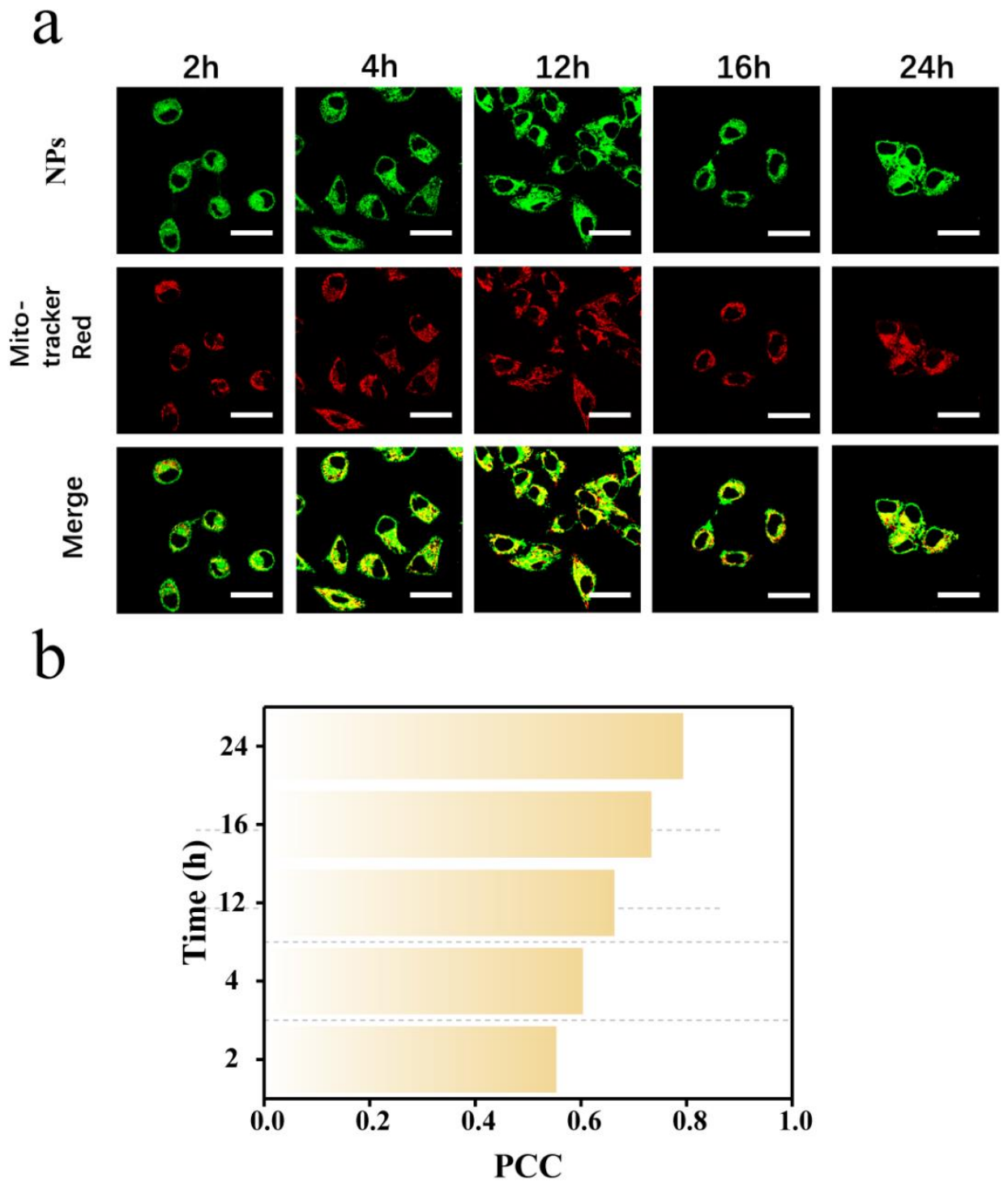

Figure S16. (a) Confocal images of HeLa cells after incubated with FITC labeled 1-FeBS-PVP colocalized with commercially mitochondria tracker at different times. Scale bar: $30 \mu \mathrm{m}$. (b) The PCC value of various groups in (a). 

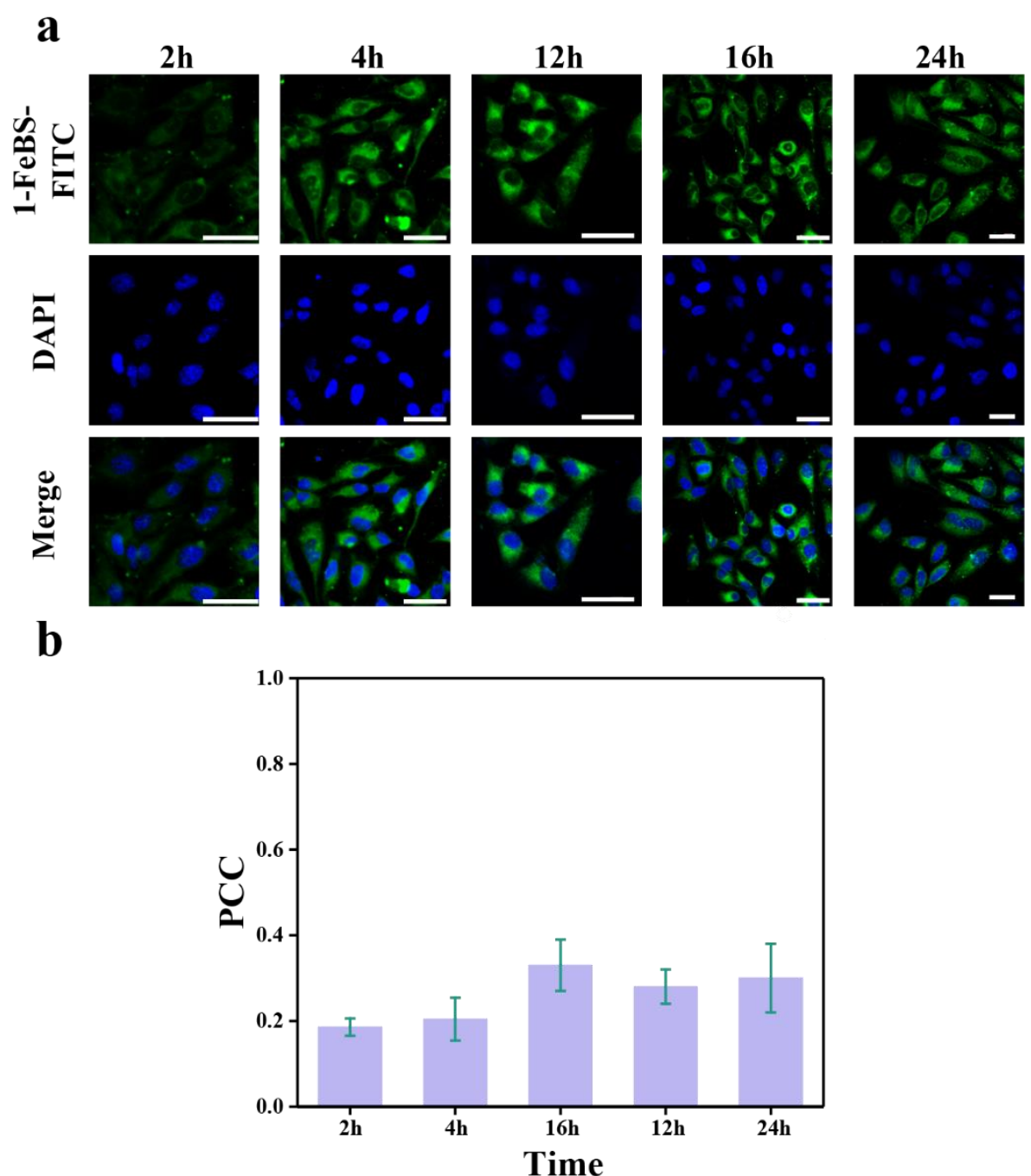

Figure S17. (a) Confocal images of HeLa cells after incubated with FITC labeled 1-FeBSPVP colocalized with commercially cell nucleus tracker at different times. Scale bar: $30 \mu \mathrm{m}$. (b) The PCC value of various groups in (a). 


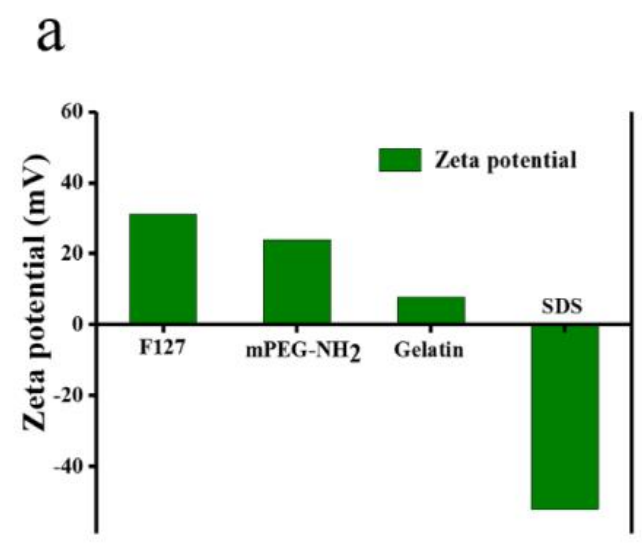

b
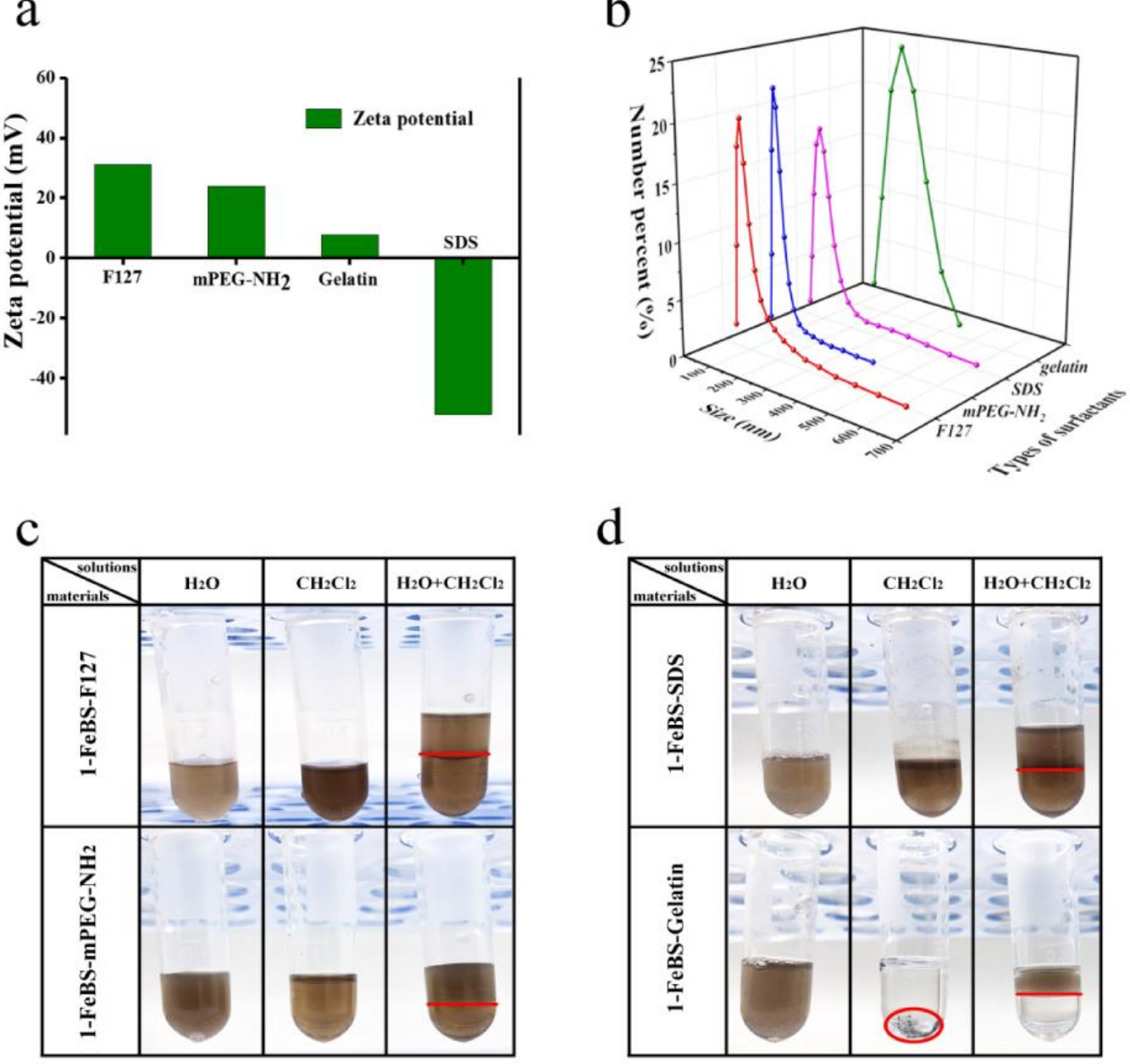

d

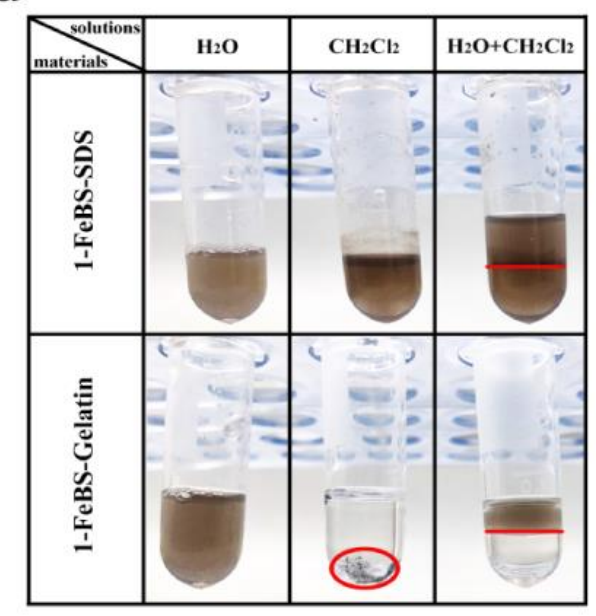

Figure S18. The characteristics with different surfactants treat. (a) Zeta potential. (b) Size distribution. (c) and (d) The solubility of 1-FeBS modified with different surfactants. Red circle: the insoluble materials. Red line: the immiscible interface of $\mathrm{H}_{2} \mathrm{O}$ and $\mathrm{CH}_{2} \mathrm{Cl}_{2}$. 


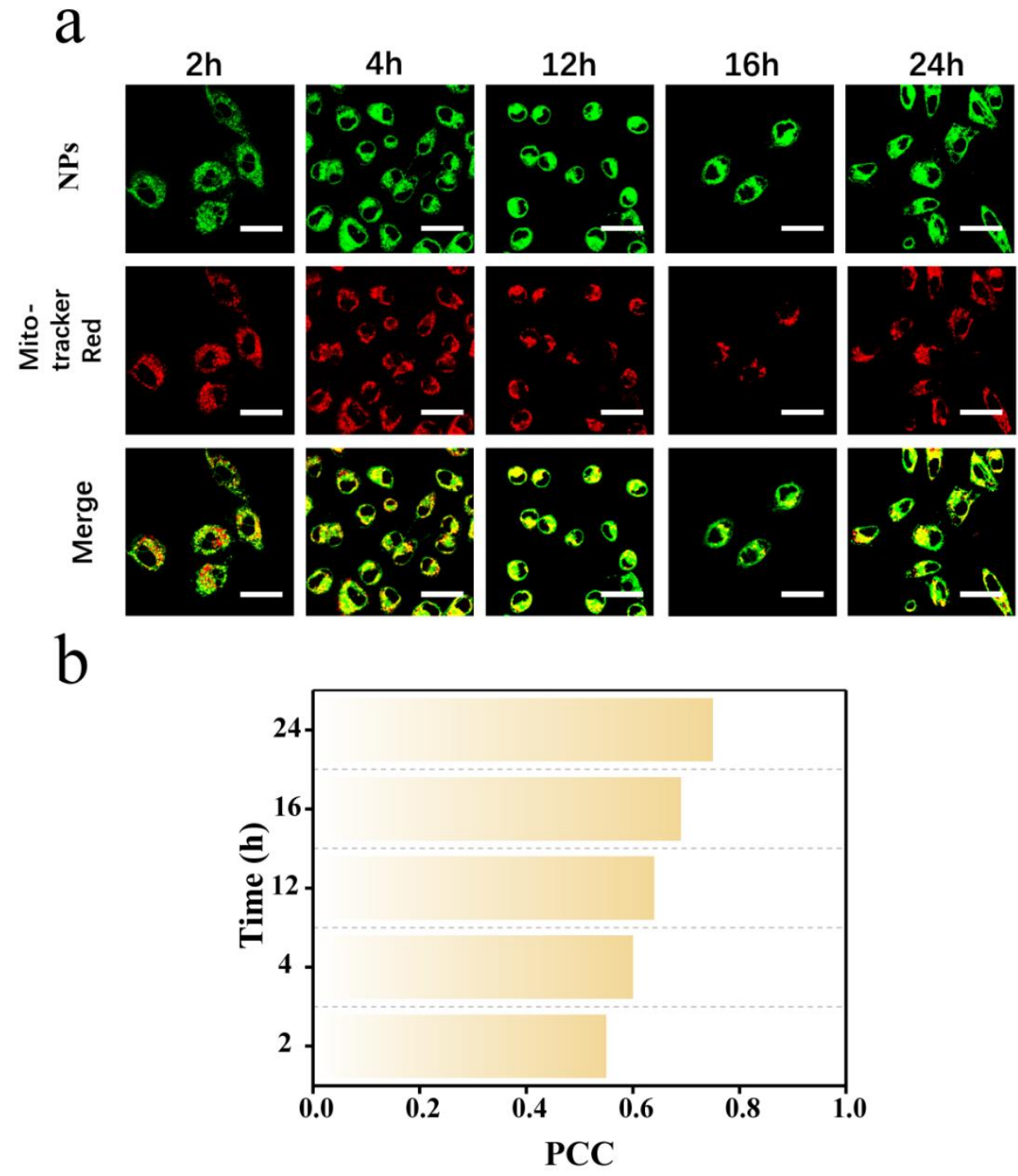

Figure S19. (a) Confocal images of HeLa cells after incubated with FITC labeled 1-FeBS-F127 colocalized with commercially mitochondria tracker at different times. Scale bar: $30 \mu \mathrm{m}$. (b) The PCC value of various groups in (a). 


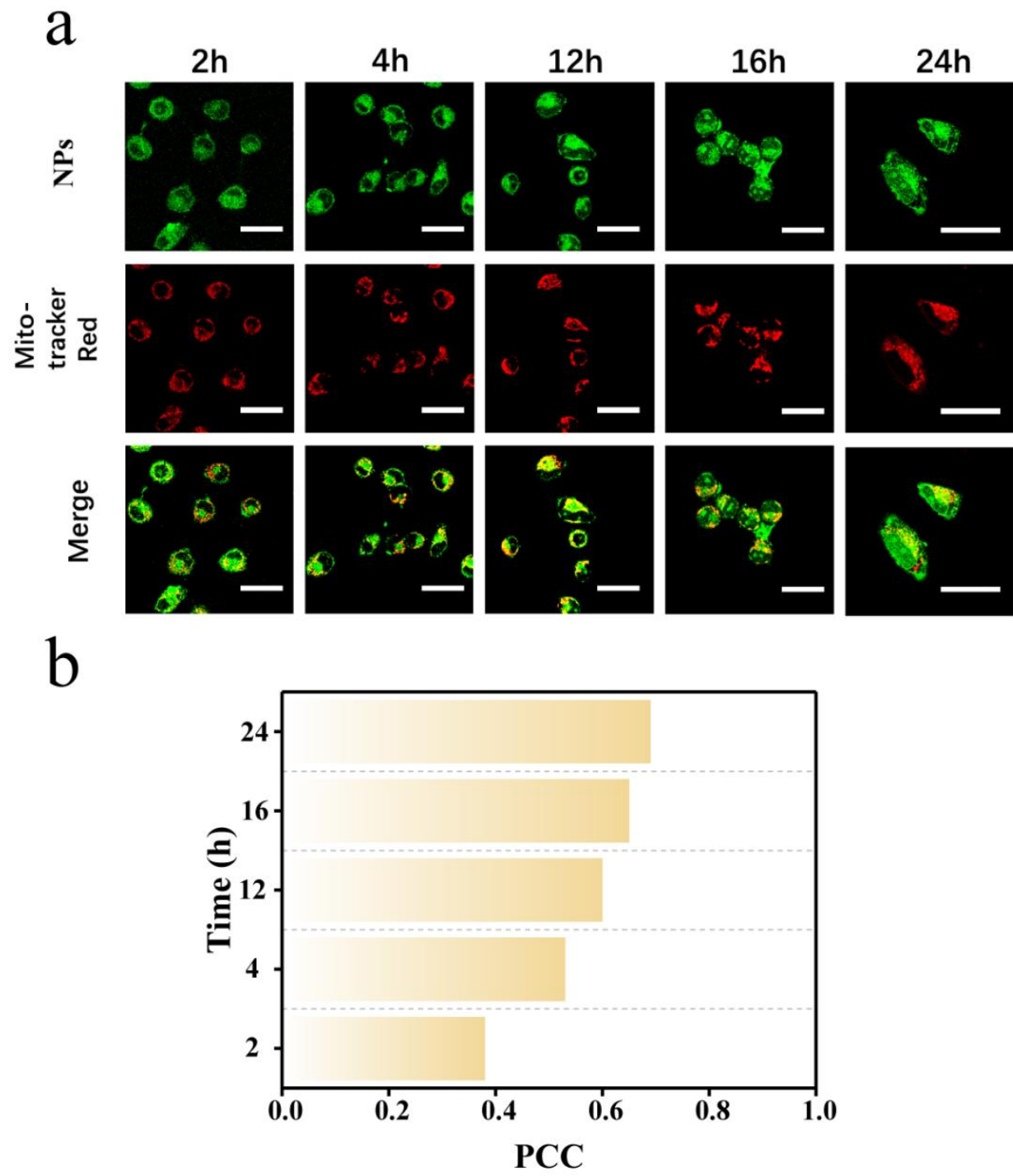

Figure S20. (a) Confocal images of HeLa cells after incubated with FITC labeled 1-FeBSmPEG-NH 2 colocalized with commercially mitochondria tracker at different times. Scale bar: $30 \mu \mathrm{m}$. (b) The PCC value of various groups in (a). 


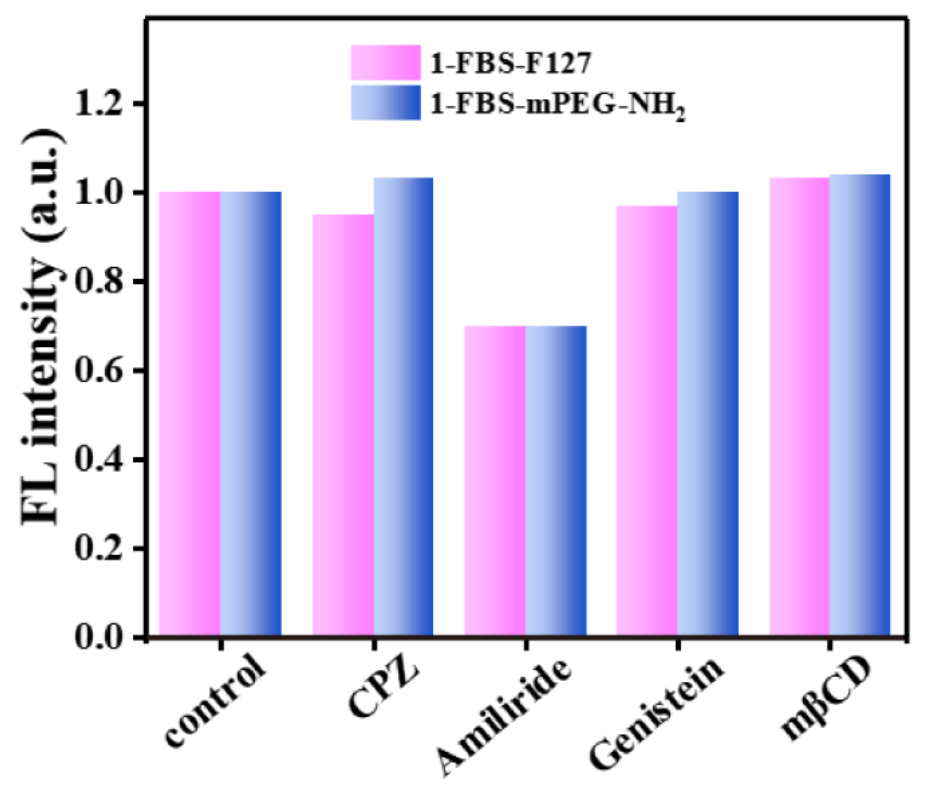

Figure S21. Cellular internalization mechanism of 1-FeBS-F127 and 1-FeBS-mPEG-NH ${ }_{2}$ in HeLa cells. The decreased FL intensity suggested that cell entry was inhibited. 


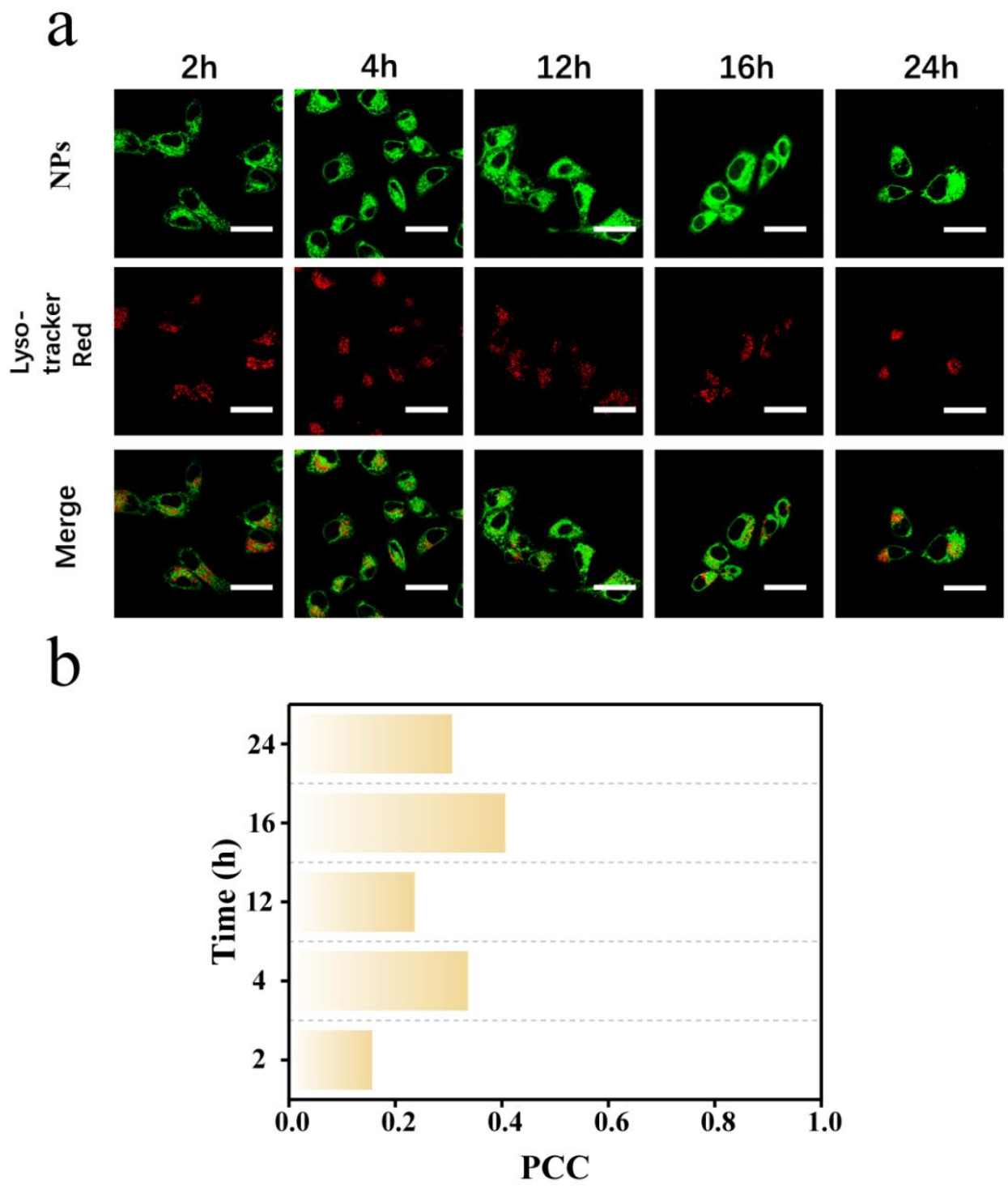

Figure S22. (a) Confocal images of HeLa cells after incubated with FITC labeled 1-FeBS-F127 colocalized with commercially lysosomes tracker at different times. Scale bar: $30 \mu \mathrm{m}$. (b) The PCC value of various groups in (a). 


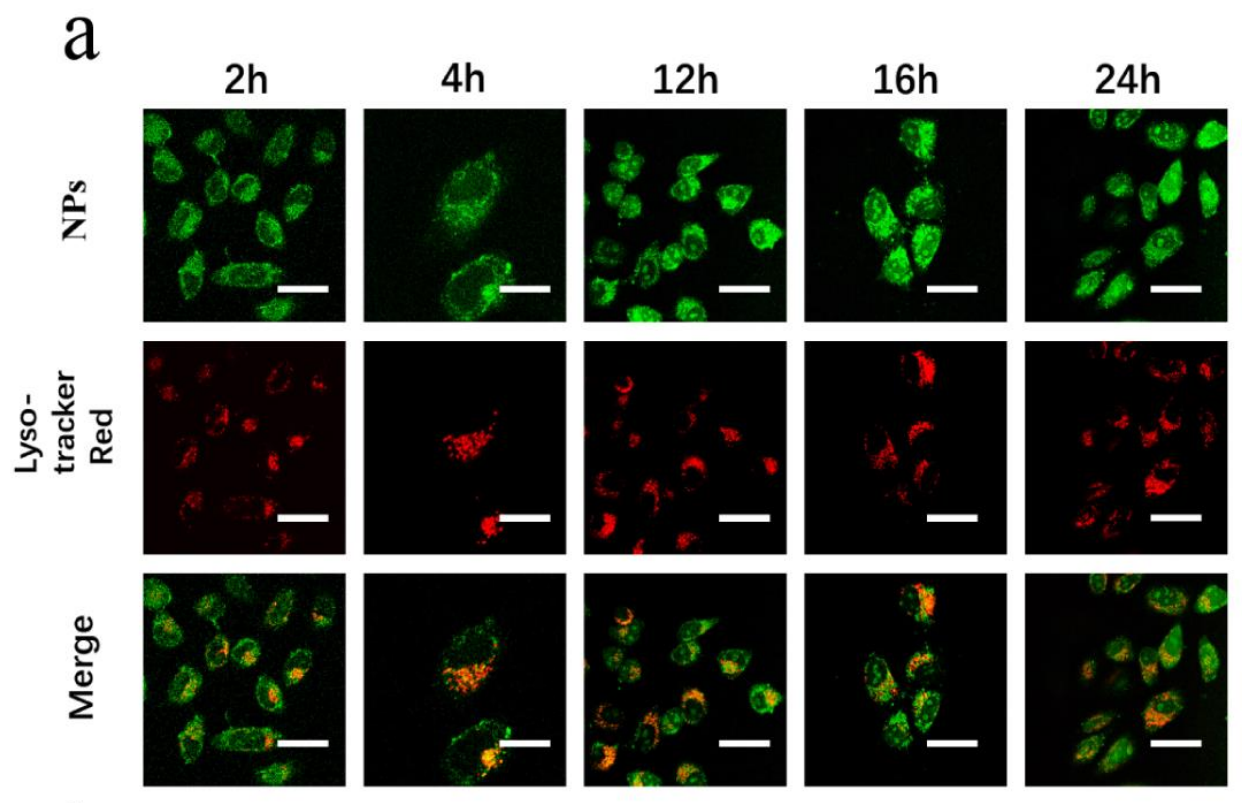

b

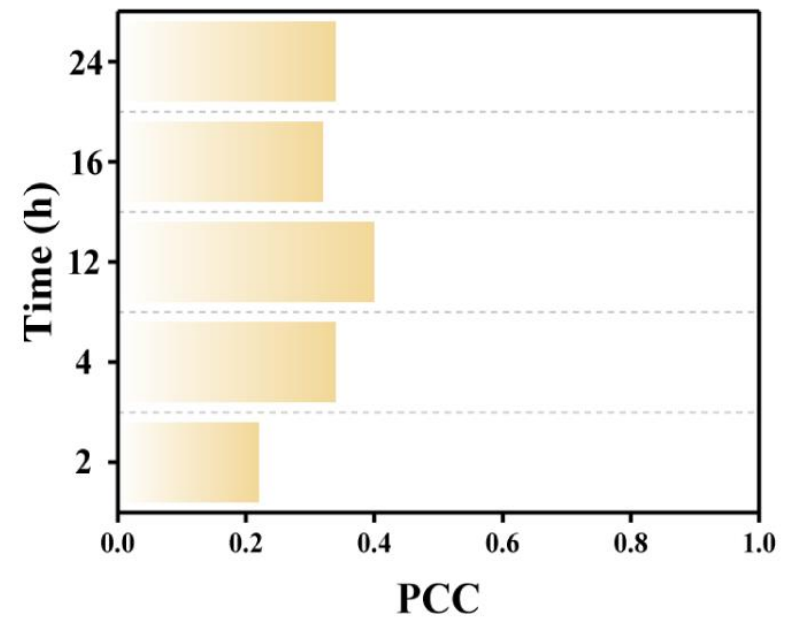

Figure S23. (a) Confocal images of HeLa cells after incubated with FITC labeled 1-FeBSmPEG-NH$H_{2}$ colocalized with commercially lysosomes tracker at different times. Scale bar: $30 \square \mu \mathrm{m}$. (b) The PCC value of various groups in (a). 


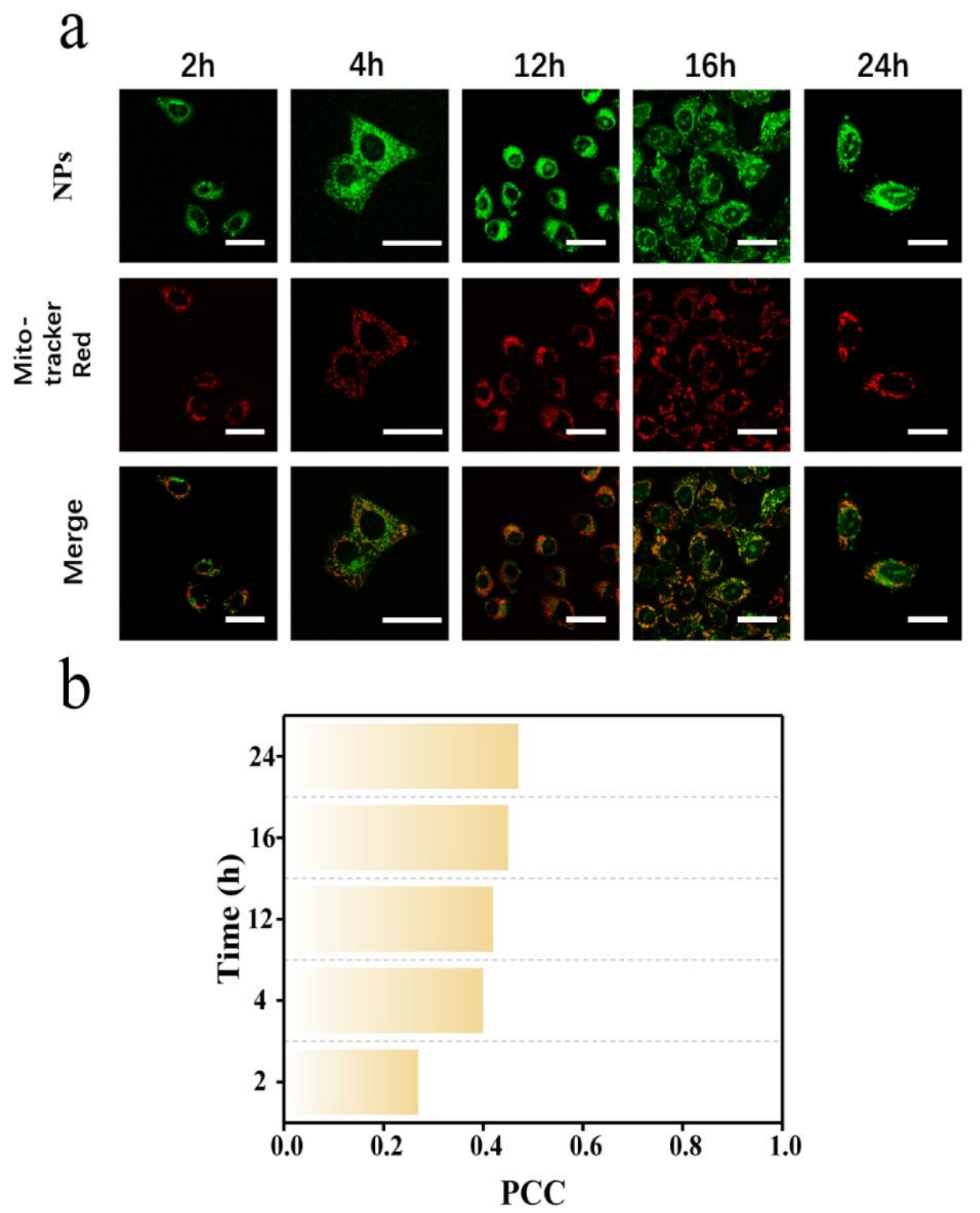

Figure S24. (a) Confocal images of HeLa cells after incubated with FITC labeled 1-FeBS-SDS colocalized with commercially mitochondria tracker at different times. Scale bar: $30 \mu \mathrm{m}$. (b) The PCC value of various groups in (a). 


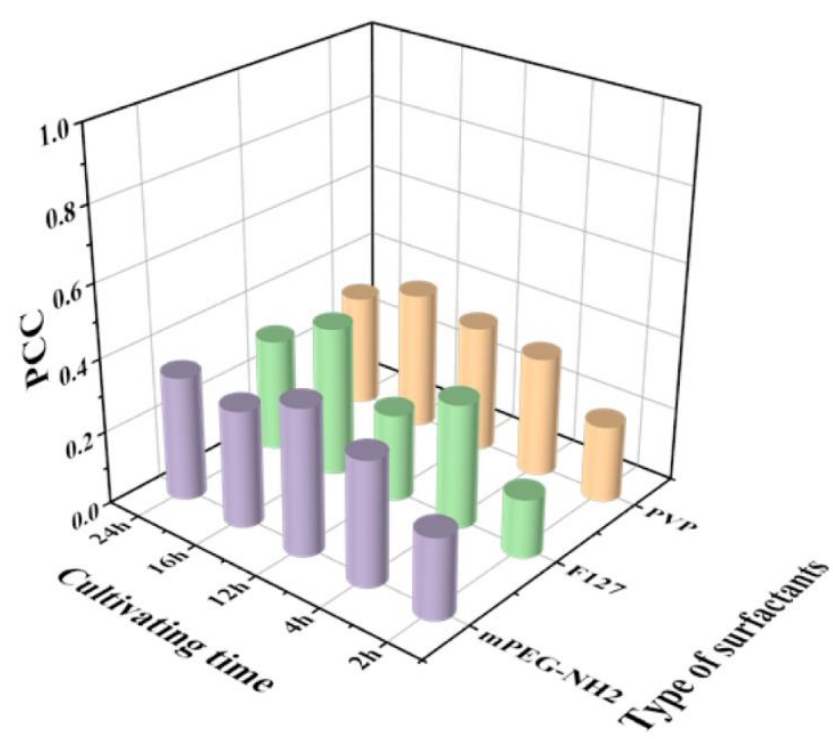

Figure S25. The PCC values of FITC-labeled 1-FeBS with different types of surfactants colocated with Lyso-tracker probe under various co-incubation times.

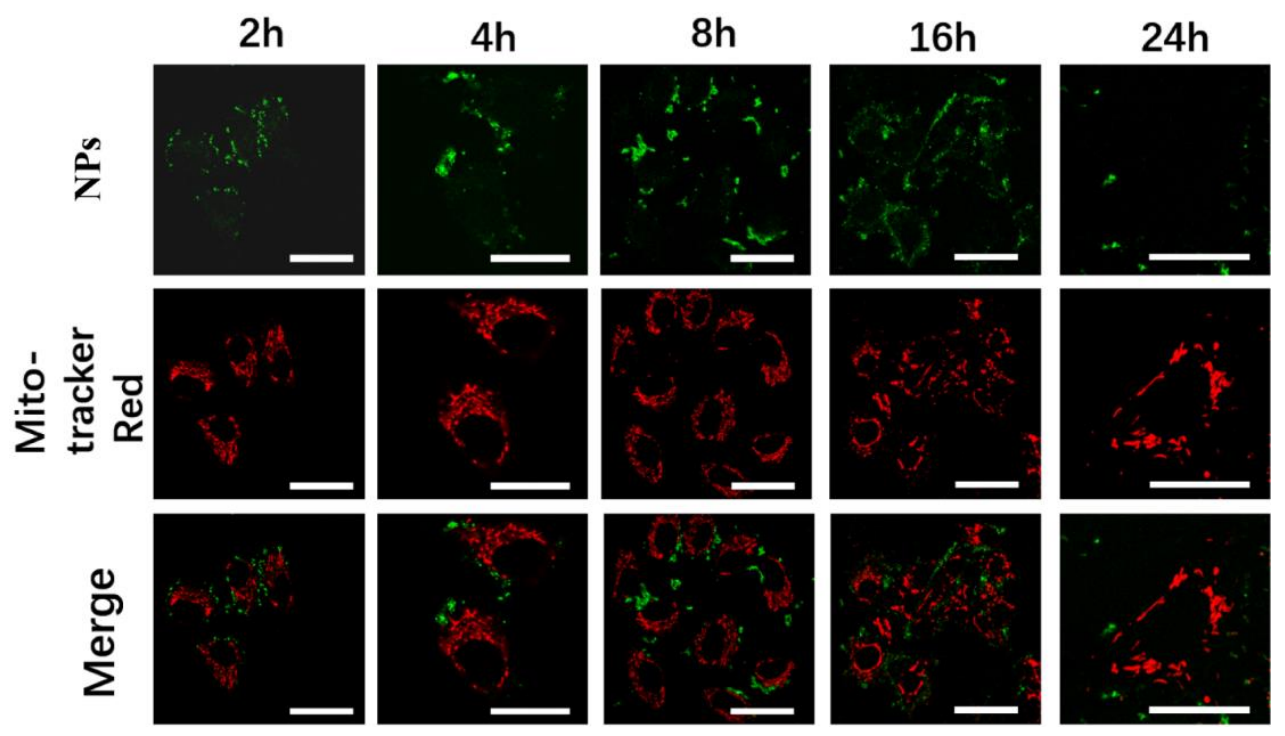

Figure S26. Confocal images of HeLa cells after incubated with FITC labeled 1-FeBS-gelatin colocalized with commercially mitochondria tracker at different times. Scale bar: $30 \mu \mathrm{m}$. 

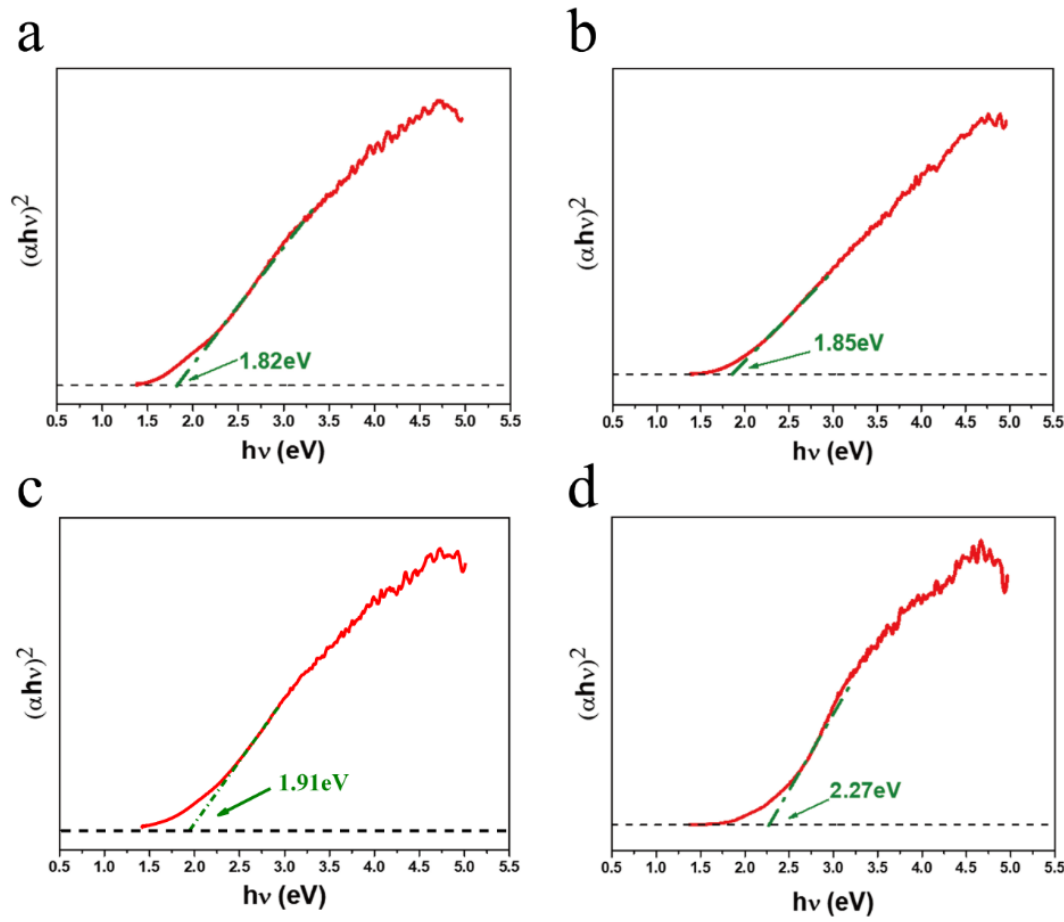

Figure S27. Tauc plots of (a) $\mathrm{Bi}_{2} \mathrm{~S}_{3}-\mathrm{PVP}$, (b) 0.5-FeBS-PVP, (c) 2-FeBS-PVP, (d) 4-FeBS-PVP derivated from the UV-vis DRS. 

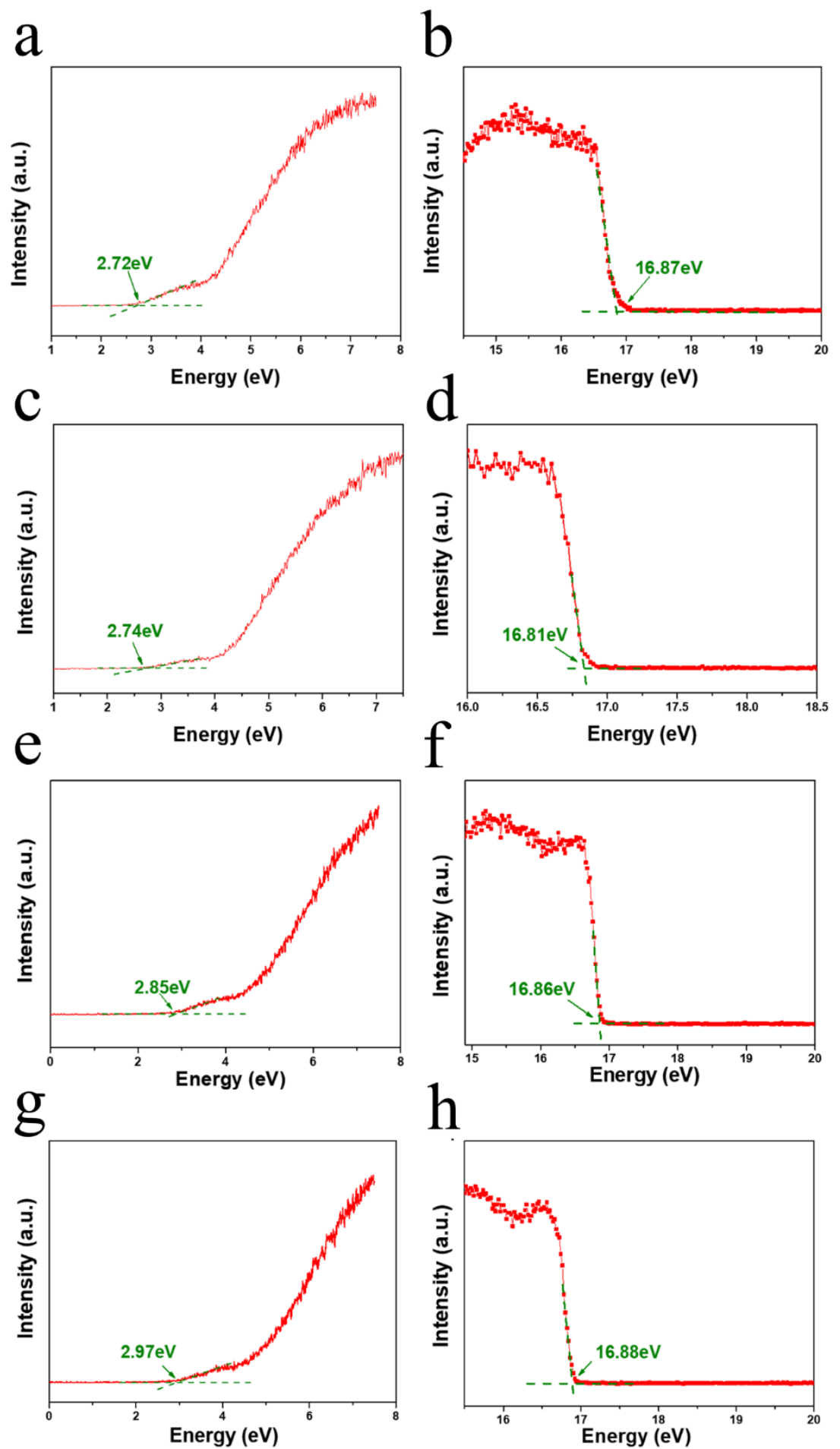

Figure S28. The UPS data of (a) and (b) $\mathrm{Bi}_{2} \mathrm{~S}_{3}-\mathrm{PVP}$, (c) and (d) 0.5-FeBS-PVP, (e) and (f) 2FeBS-PVP, (g) and (h) 4-FeBS-PVP. 
a

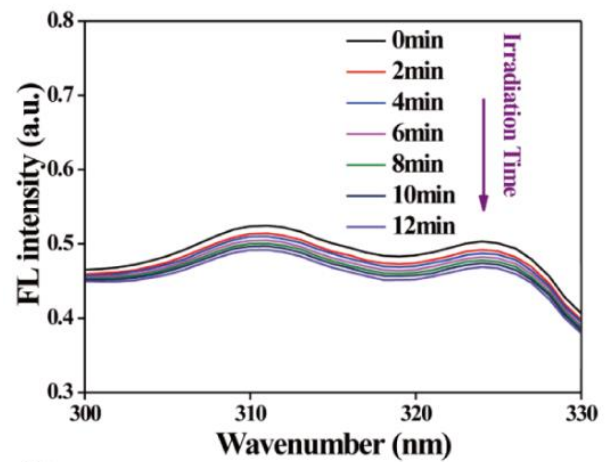

C

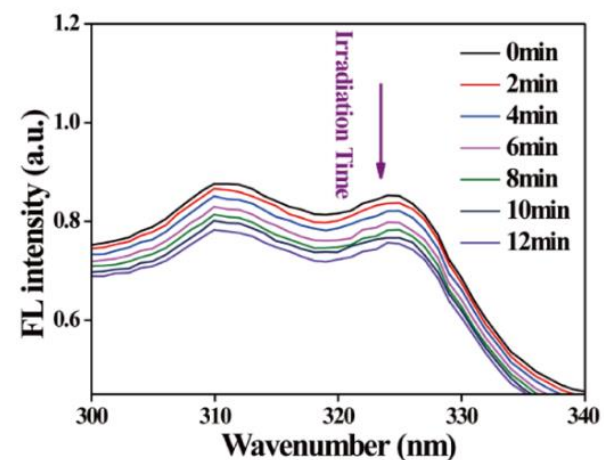

e

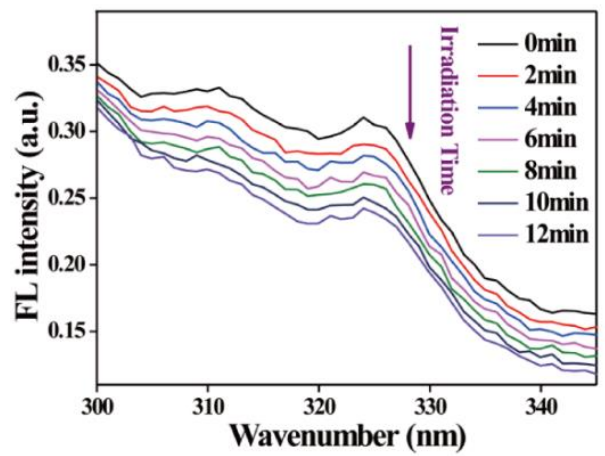

b

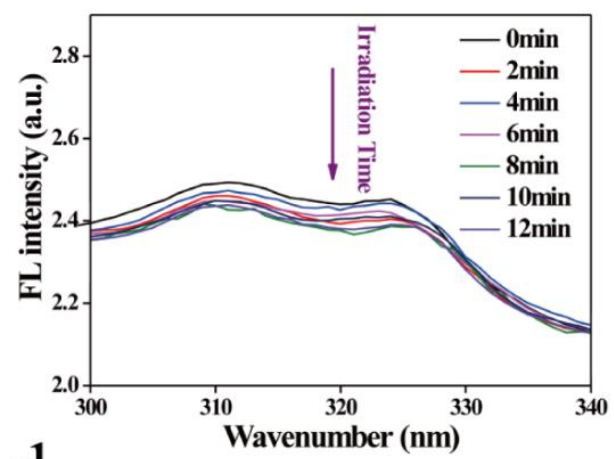

d

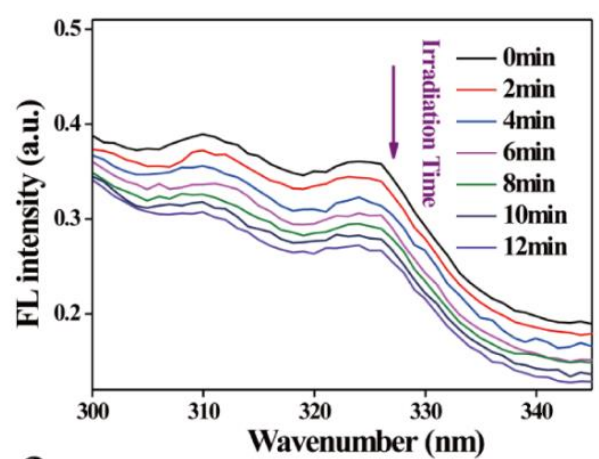

f

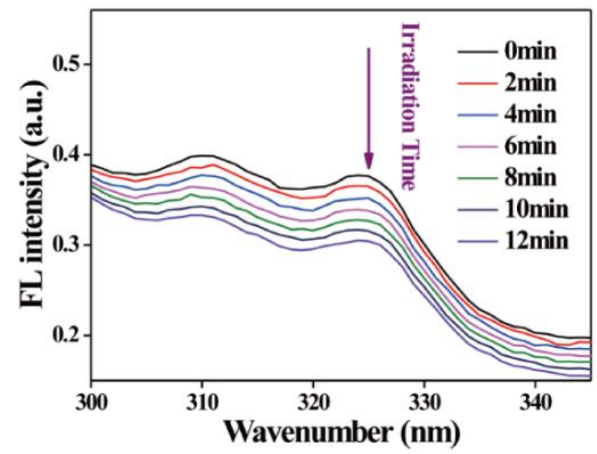

Figure S29. The decrease in fluorescence of DPBF after irradiated by $650 \mathrm{~nm}$ laser and recorded every 2 minutes, showing the ability to produce single oxygen. (a) DPBF. (b) DPBF $+\mathrm{Bi}_{2} \mathrm{~S}_{3}$. (c) DPBF + 0.5-FeBS-PVP. (d) DPBF + 1-FeBS-PVP. (e) DPBF + 2-FeBS-PVP. (f) DPBF + 4FeBS-PVP. 


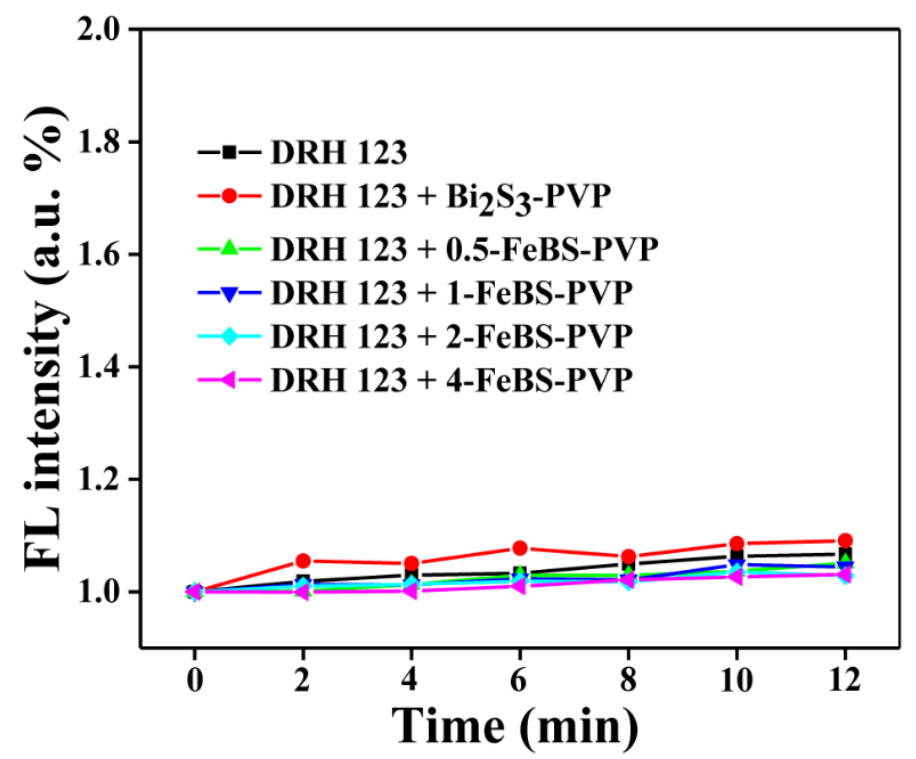

Figure S30. Ability to produce $\mathrm{O}_{2}{ }^{--}$of $x$-FeBS-PVP. 
a

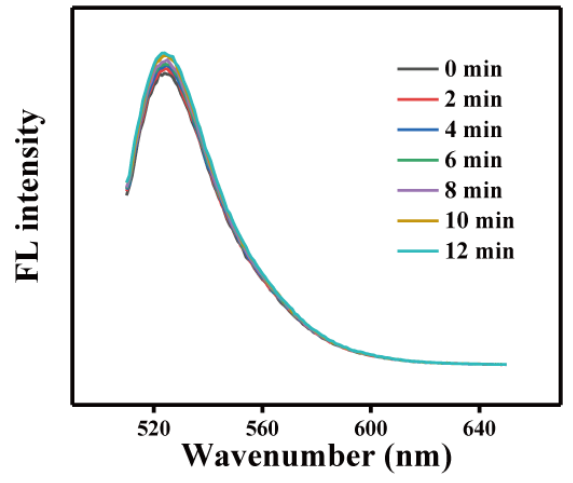

C

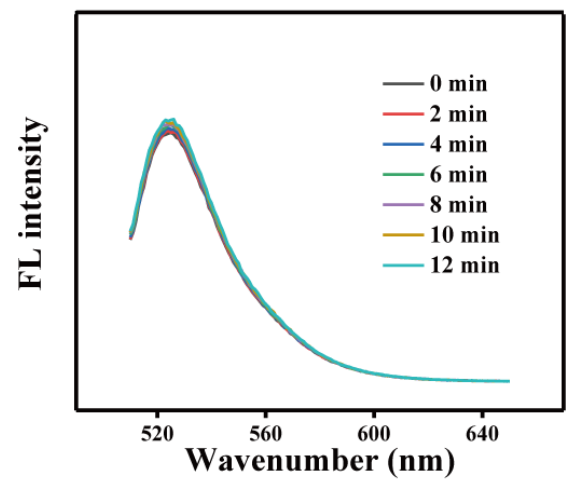

e

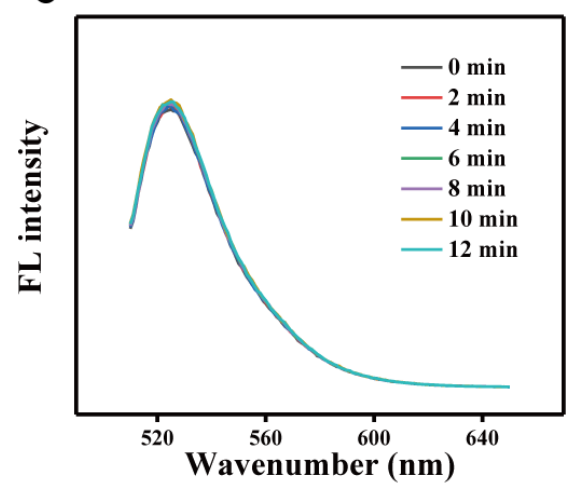

b

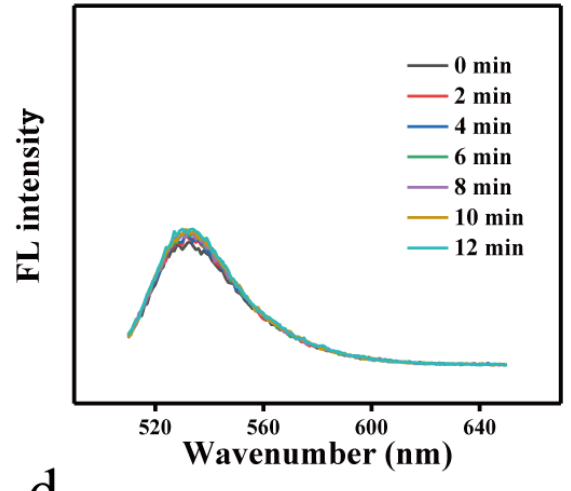

d

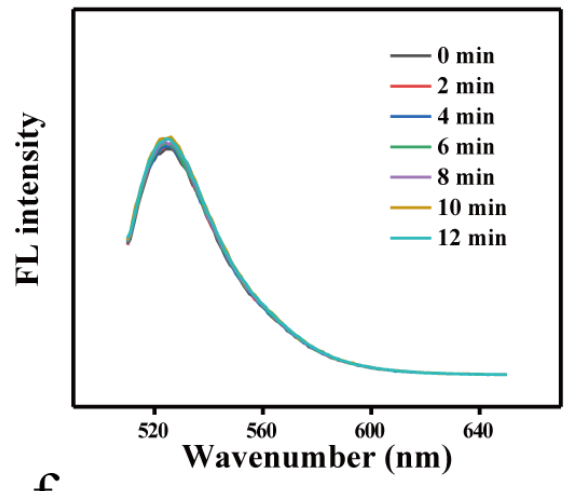

$\mathrm{f}$

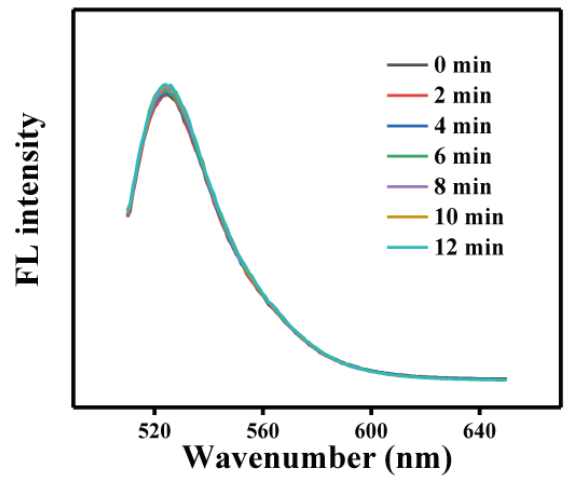

Figure S31. The fluorescence intensity changes of different groups after irradiated by $650 \mathrm{~nm}$ laser and recorded every 3 minutes. (a) DRH123, (b) DRH123+Bi $2 \mathrm{~S}_{3}-\mathrm{PVP}$, (c) DRH123+0.5FeBS-PVP, (d) DRH123+1-FeBS-PVP, (e) DRH123+2-FeBS-PVP, (f) DRH123+4-FeBS-PVP. 

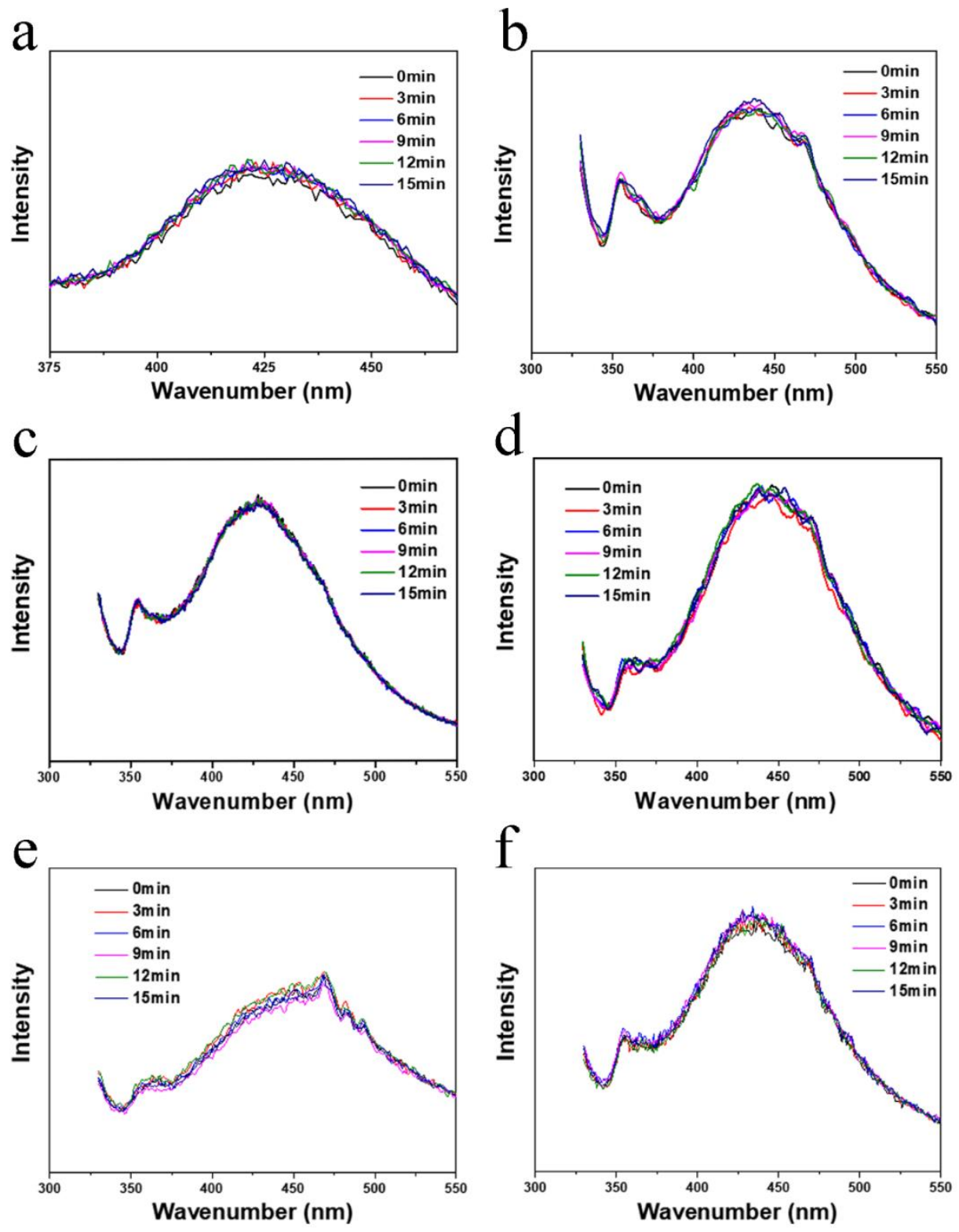

Figure S32. The changes in fluorescence of TA after irradiated by $650 \mathrm{~nm}$ laser and recorded every 3 minutes, indicating the power of generating hydroxyl radicals. (a) TA, (b) TA+Bi $\mathrm{S}_{3}-$ PVP, (c) TA+0.5-FeBS-PVP, (d) TA+1-FeBS-PVP, (e) TA+ 2-FeBS-PVP, (f) TA+4-FeBSPVP. 
a
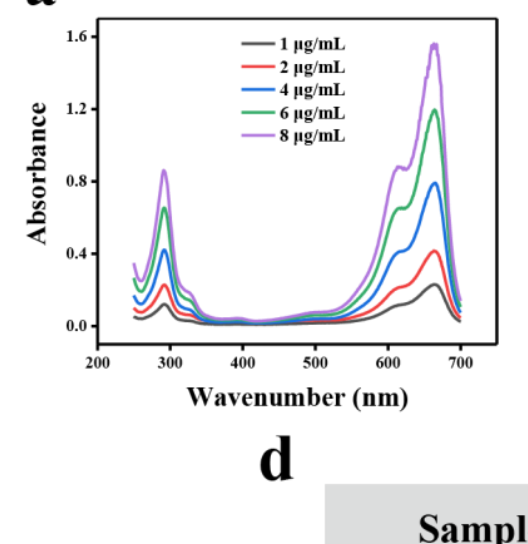

0.5-FeBS-PVP

1-FeBS-PVP

2-FeBS-PVP

4-FeBS-PVP b

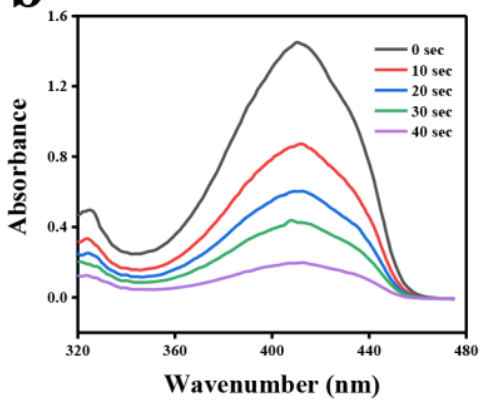

c

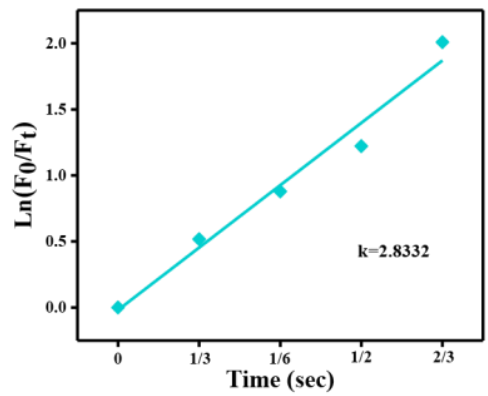

Absorption at $650 \mathrm{~nm}$

$\Phi_{\Delta}$

$0.5057 \quad 0.19 \%$

0.4107

$0.48 \%$

0.7112

$0.37 \%$

0.1576

$0.49 \%$

Figure S33 (a) The absorbance of MB in different concentrations. (b) Degradation of singlet oxygen by methylene blue. (c) Degradation kinetics of DPBF by methylene blue. (d) The calculated singlet oxygen quantum yield of different samples.

As shown in Figure 3d, the degradation capacity of DPBF is ranked as follows: 1-FeBSPVP > 2-FeBS-PVP > 4-FeBS-PVP > 0.5-FeBS-PVP. The degradation kinetics of DPBF was obtained in Figure 3f, where F0 and Ft are the characteristic absorbance of DPBF before and after irradiation, respectively. Methylene blue (MB) solution was used as a standard reference, with the singlet oxygen yield of $0.52 .{ }^{1}$ The degradation capacity of MB was also measured and shown in Figure S33. The singlet oxygen quantum yield was calculated by the following formula:

$$
\varphi_{\Delta(X)}=\varphi_{\Delta(s t d)}\left(\frac{S_{X}}{S_{s t d}}\right)\left(\frac{F_{s t d}}{F_{X}}\right)
$$

where subscript $X$ and std represent the sample and MB, respectively. $S$ is the slope of the plot of degradation kinetics. $F$ is the absorption correction factor. ${ }^{2}$ 

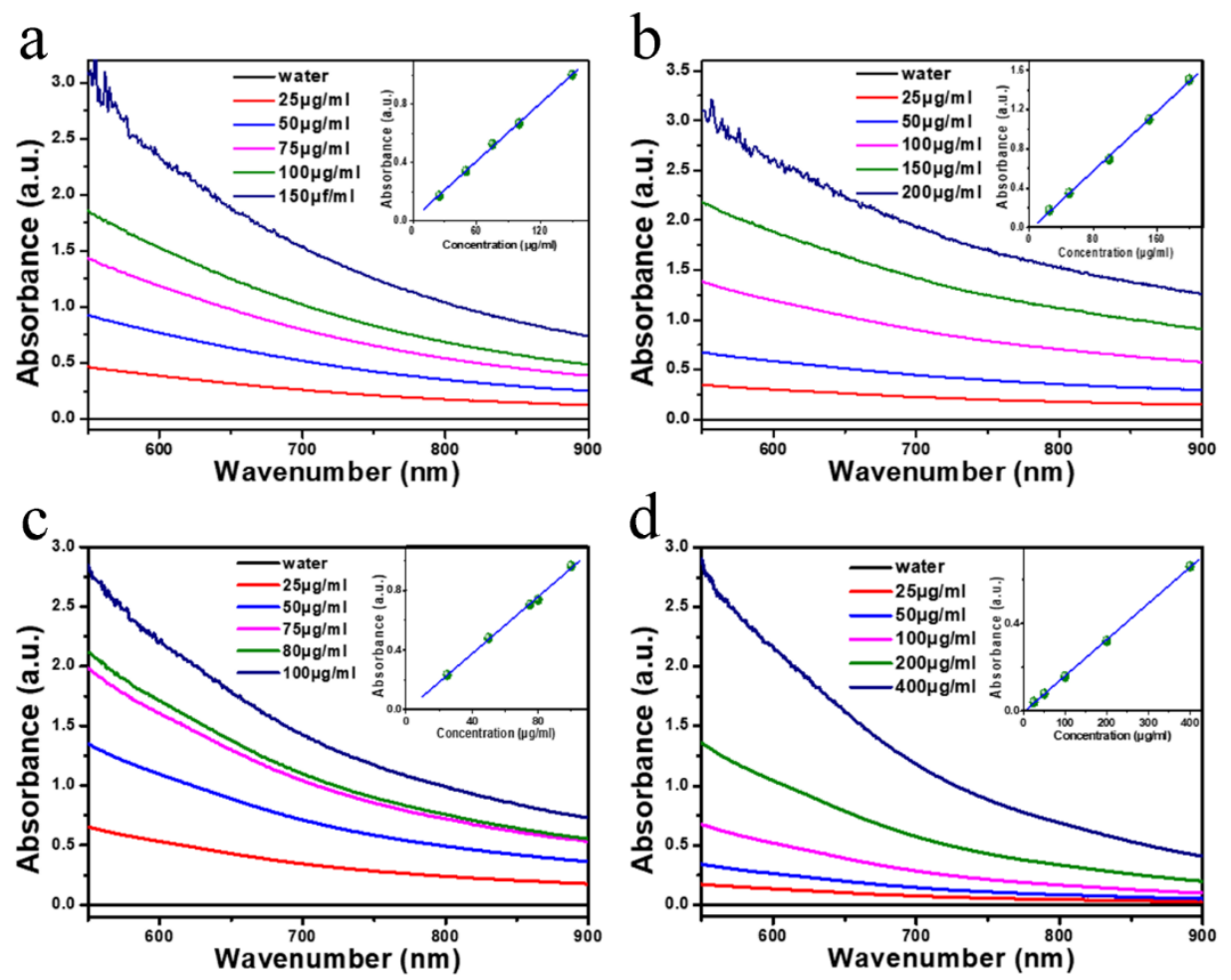

Figure S34. UV-vis absorption spectral of (a) 0.5-FeBS, (b) 1-FeBS, (c) 2-FeBS, (d) 4-FeBS at different concentrations. Insert: the standard curve of the Absorbance-Concentration at 808 nm. 

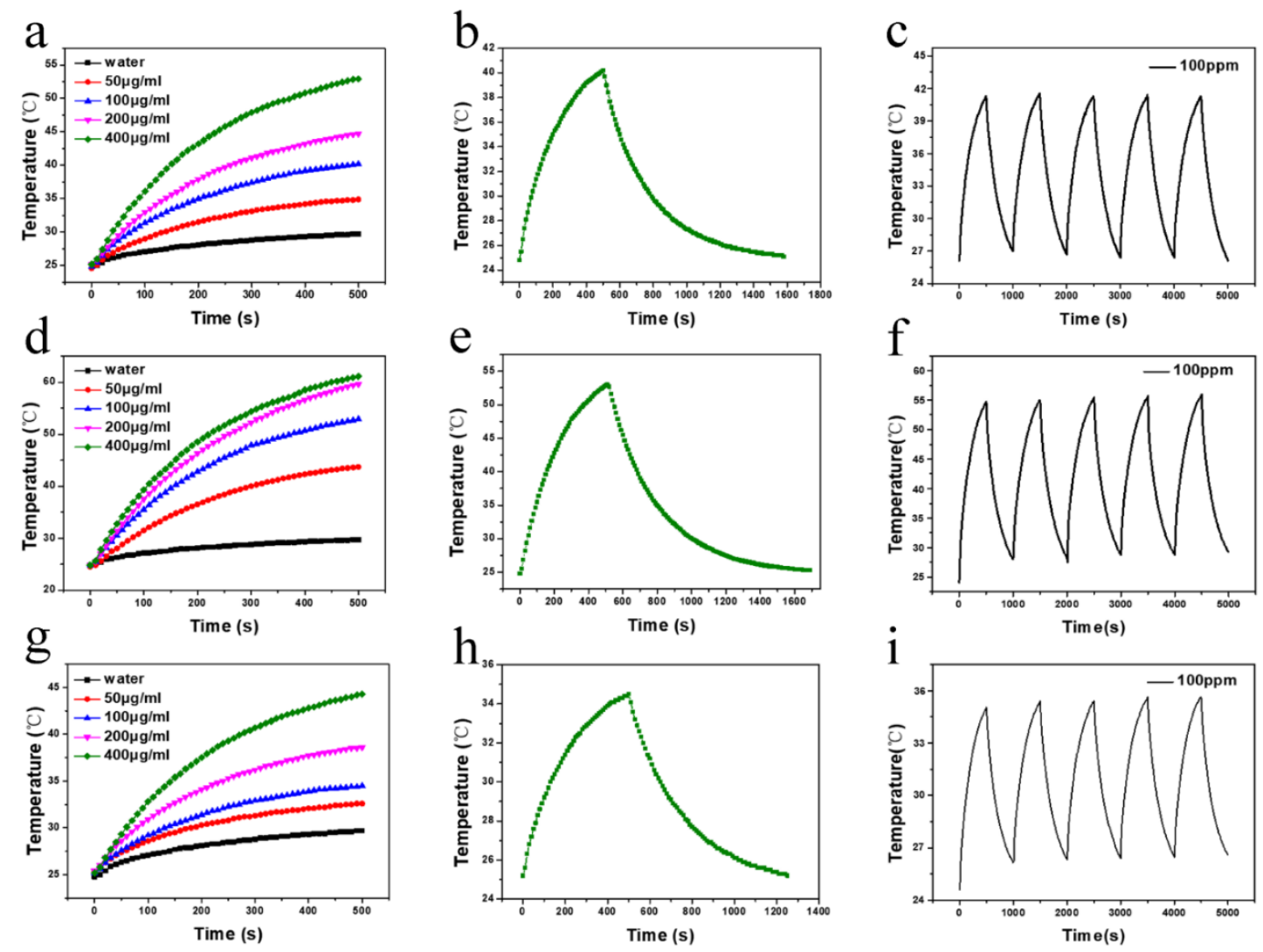

Figure S35. The photothermal effect irradiated by $808 \mathrm{~nm}$ laser $\left(2 \mathrm{~W} / \mathrm{cm}^{2}\right)$. The temperature changes of (a) 0.5-FeBS-PVP, (d) 2-FeBS-PVP, (g) 4-FeBS-PVP at different concentrations irradiated for $500 \mathrm{~s}$. The cycles involving irradiation for $500 \mathrm{~s}$ and followed by passive cooling of (b) 0.5-FeBS-PVP, (e) 2-FeBS-PVP, (h) 4-FeBS-PVP. Photostability curve recording irradiated for 500s followed by cooling down for serval cycles of (c) 0.5 -FeBS-PVP, (f) 2FeBS-PVP, (i) 4-FeBS-PVP. 
a

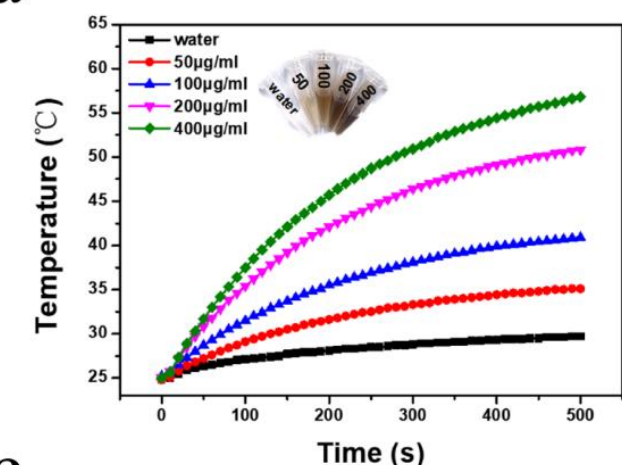

$\mathrm{C}$

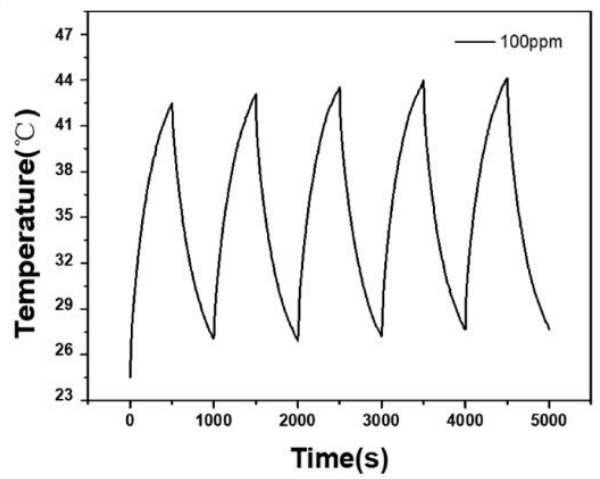

b

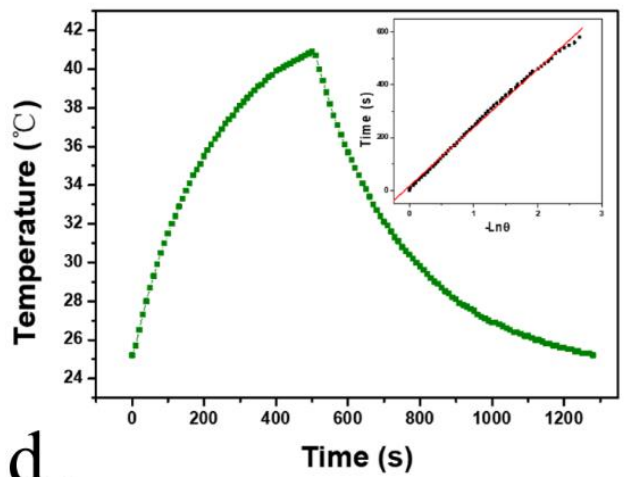

d

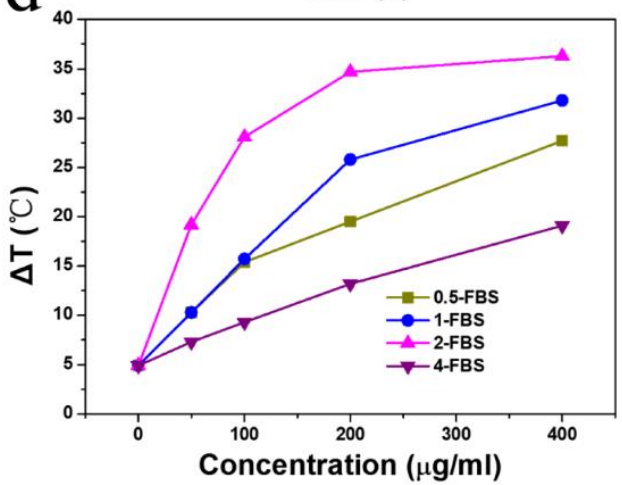

Figure S36. The photothermal effect of 1-FeBS-PVP, irradiated by $808 \mathrm{~nm}$ laser $\left(2 \mathrm{~W} / \mathrm{cm}^{2}\right)$. (a) The temperature changes. Insert: photograph of aqueous dispersions of 1-FeBS-PVP at different concentrations. (b) Cooling curve after irradiated for $500 \mathrm{~s}$ and then stopped. Insert: time versus $-\ln \theta$ curve to calculate the photothermal conversion efficiency. (c) Photostability curve, recording irradiated for $500 \mathrm{~s}$ followed by cooling down for serval cycles. (d) The temperature changes of $x$-FeBS-PVP at different concentrations under $500 \mathrm{~s}$ of irradiation. 

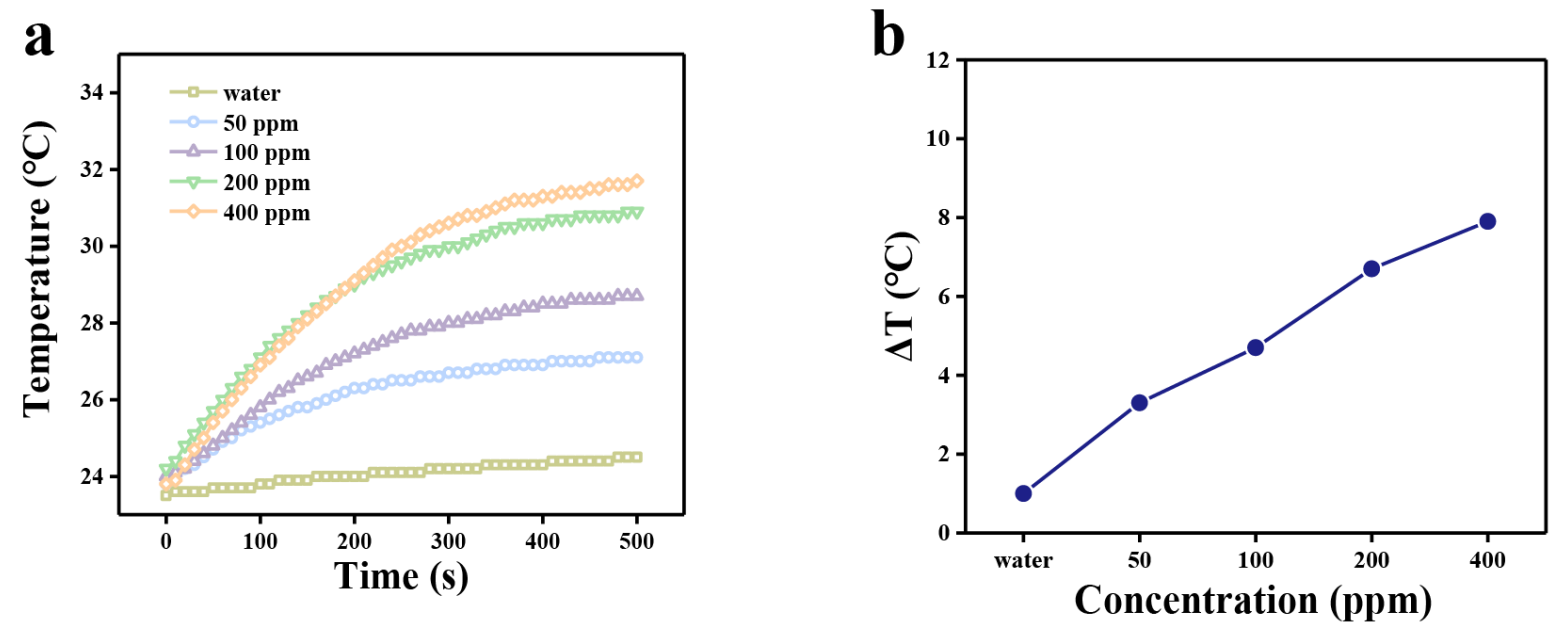

c

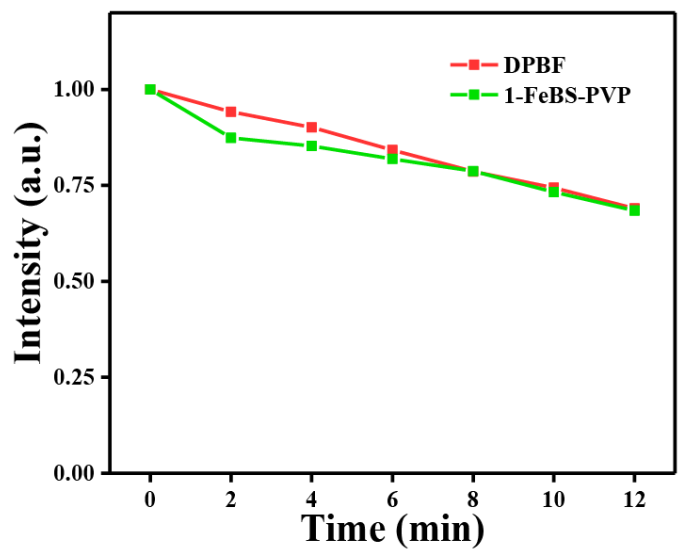

Figure S37. (a) The temperature changes of 1-FeBS-PVP solution under the irradiation of 650 nm laser. (b) The $\Delta \mathrm{T}$ of different concentrations at 500s. (c) The ability of 1-FeBS-PVP to degrade DPBF under the irradiation of $808 \mathrm{~nm}$ laser. 

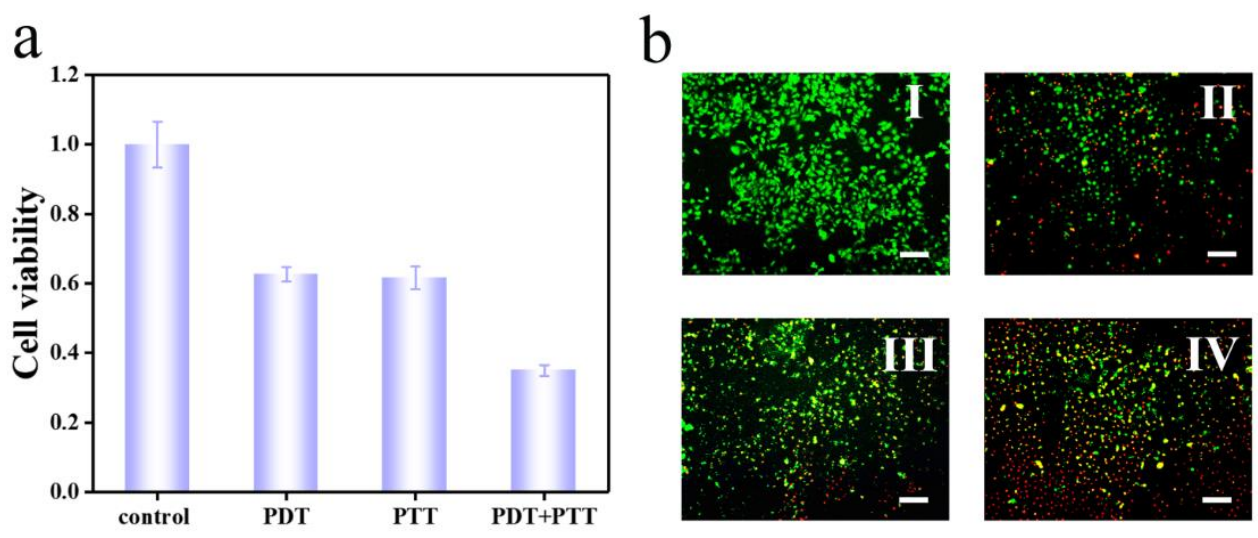

Figure S38. Cytotoxicity after different treatment. (a) The cell viability was investigated by MTT assay. (b) Fluorescence images of calcein-AM and PI labeled HeLa cells. (I) control, (II) PDT, (III) PTT, (IV) PDT\&PTT. Scale bar: $250 \mu \mathrm{m}$.
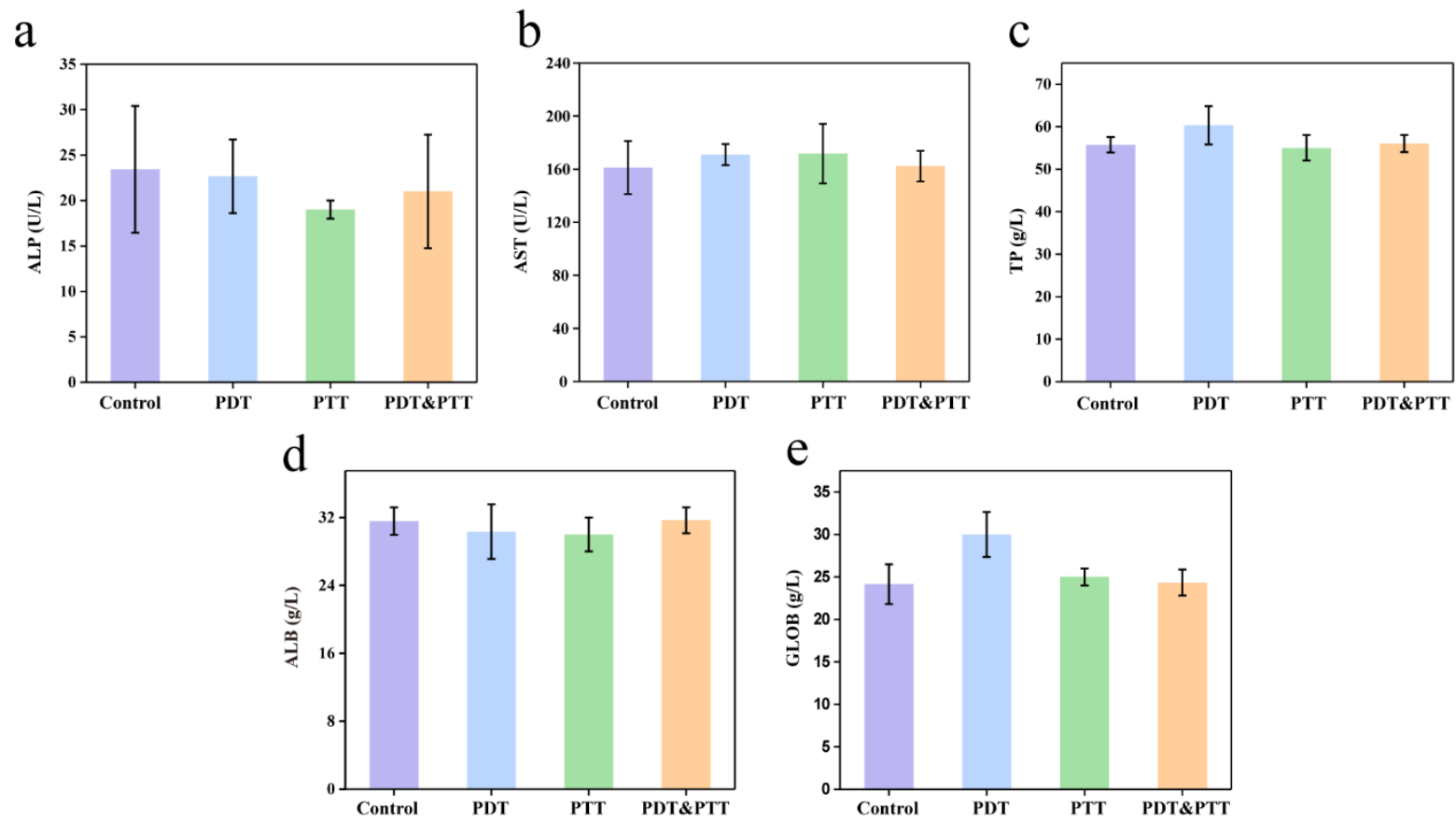

Figure S39. The liver function indexes of mice after various treatments. (a) Alkaline phosphatase (ALP), (b) Aspartate aminotransferase (AST), (c) Total protein (TP), (d) Albumin (ALB), (e) Globulin (GLOB). 

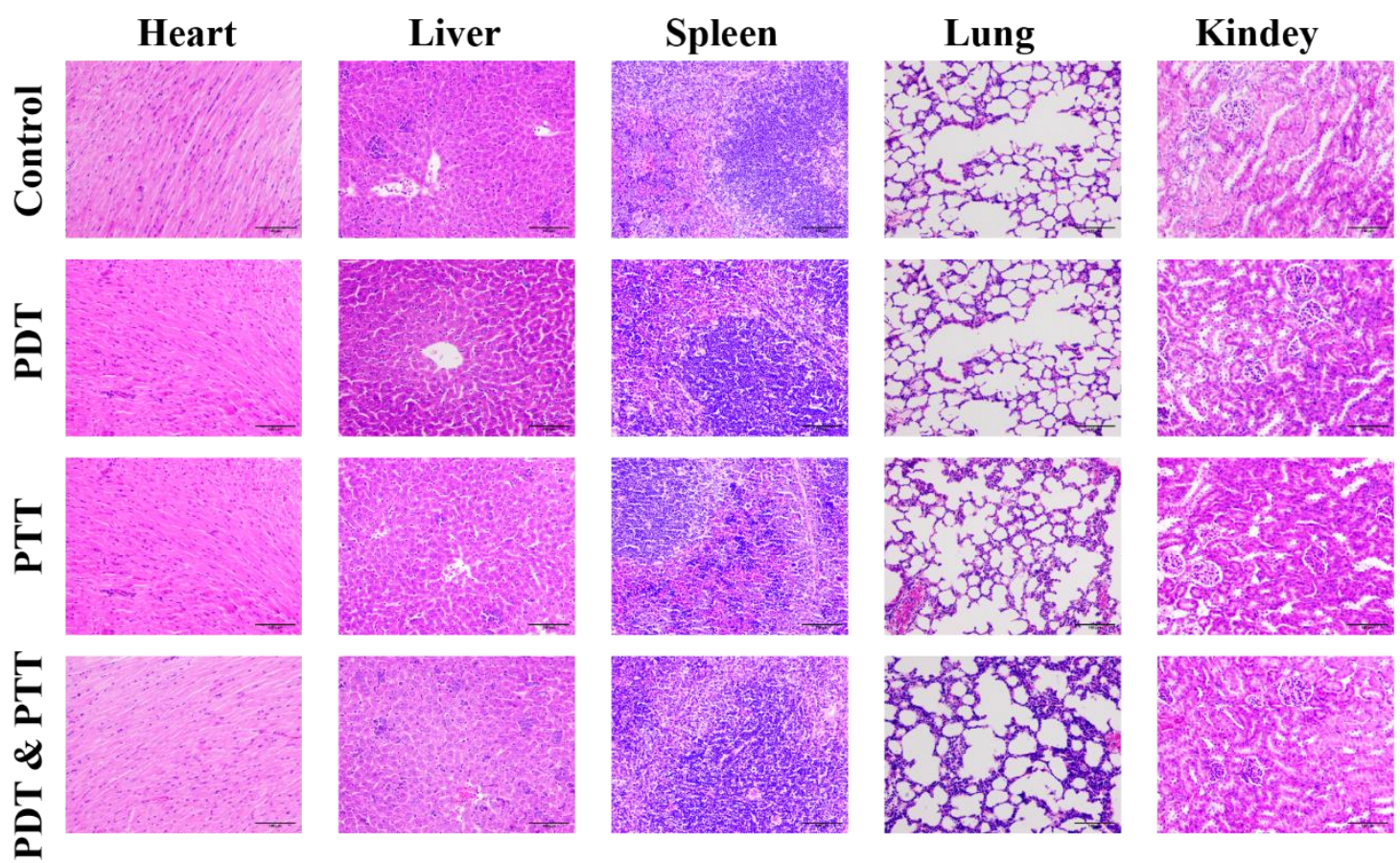

Figure S40. H\&E staining images of major organs collected from tumor-bearing mice after

14 days of treatment. Scale bar: $100 \mu \mathrm{m}$.

\section{REFERENCES}

(1) Redmond R. W.; Gamlin, J. N. A Compilation of Singlet Oxygen Yields from Biologically Relevant Molecules. Photochem. Photobiol., 1999, 70, 391-475.

(2) Qian, C.; Feng, P.; Yu, J.; Chen, Y.; Hu, Q.; Sun, W.; Xiao, X.; Hu, X.; Bellotti, A.; Shen, Q.; Gu, Z. Anaerobe-Inspired Anticancer Nanovesicles. Angew. Chem. Int. Ed. 2017, 56, $2588-2593$. 